\title{
Feasibility and efficacy of mass testing for SARS- CoV-2 in a UK university using swab pooling and PCR
}

\section{Ben Warne ( $\sim$ bw252@cam.ac.uk)}

University of Cambridge https://orcid.org/0000-0003-1326-0373

Jessica Enright

University of Glasgow https://orcid.org/0000-0002-0266-3292

\section{Marina Metaxaki}

University of Cambridge, Department of Medicine, Cambridge, UK

\section{Stewart Fuller}

University of Cambridge, Department of Medicine, Cambridge, UK

\section{Richard J. Samworth}

Statistical Laboratory, Centre for Mathematical Sciences, University of Cambridge

\section{Gillian Weale}

Health, Safety \& Regulated Facilities Division, University of Cambridge, Cambridge, UK

Jon Holgate

University Information Services, University of Cambridge, Cambridge, UK

\section{Vijay Samtani}

University of Cambridge https://orcid.org/0000-0002-4246-6453

\section{Craig Brierley}

Office of External Affairs and Communications, University of Cambridge

\section{Dinesh Aggarwal}

University of Cambridge https://orcid.org/0000-0002-5938-8172

\section{Sarah Hilborne}

University of Cambridge

\section{Mahin Bagheri Kahkeshi}

University of Cambridge

\section{Sarah Berry}

University of Cambridge

Julie A. Douthwaite

Biopharma RD, AstraZeneca

\author{
Alexandra L. Orton \\ https://orcid.org/0000-0002-5440-6802
}

\section{Rebecca Clarke}


Institute for Manufacturing, Department of Engineering, University of Cambridge

\section{Darius Danaei}

Institute for Manufacturing, Department of Engineering, University of Cambridge

\section{Rory Dyer}

Institute for Manufacturing, Department of Engineering, University of Cambridge

\section{Rob Glew}

Institute for Manufacturing, Department of Engineering, University of Cambridge

\section{Oliver Lambson}

Institute for Manufacturing, Department of Engineering, University of Cambridge

\section{Aastha Dahal}

Cambridge Students' Union

\section{Ben Margolis}

Cambridge Students' Union

\section{Edna Murphy}

Office of Intercollegiate Services Ltd., Cambridge

\section{Matthew Russell}

Office of Intercollegiate Services Ltd., Cambridge

\section{Vickie S. Braithwaite}

Public Health Directorate, Cambridgeshire County Council and Peterborough City Council

\section{Kathryn Faulkner}

Public Health Directorate, Cambridgeshire County Council and Peterborough City Council

\section{Elizabeth Wright}

Public Health Directorate, Cambridgeshire County Council and Peterborough City Council, UK

\section{Afzal Chaudhry}

Cambridge Institute for Medical Research https://orcid.org/0000-0001-6156-9194

\section{Lenette Mactavous}

Cambridge University Hospitals NHS Foundation Trust

\section{Maharshi Dhada}

Institute for Manufacturing, Department of Engineering

\section{Ajith Parlikad}

Institute for Manufacturing, Department of Engineering

University of Cambridge Asymptomatic COVID-19 Screening Programme Consortium

University of Cambridge (see Supplementary File for full list of authors)

\section{Jane Greatorex}

Lucy Cavendish College, University of Cambridge

\section{Ian Leslie}

University Information Services, University of Cambridge

\section{Andy Neely}

Vice Chancellor's Office, University of Cambridge 


\section{Steve Rees}

Biopharma RD, AstraZeneca

\section{Ashley Shaw}

Cambridge University Hospitals NHS Foundation Trust

\section{Martin Vinnell}

Occupational Health and Safety Services, University of Cambridge

\section{Linda Sheridan}

Public Health Directorate, Cambridgeshire County Council and Peterborough City Council

\section{Emmeline Watkins}

Public Health Directorate, Cambridgeshire County Council and Peterborough City Council, UK

\section{Stephen Baker}

University of Cambridge https://orcid.org/0000-0003-1308-5755

\section{lan G. Goodfellow}

University of Cambridge

Paul J. Lehner

University of Cambridge https://orcid.org/0000-0001-9383-1054

\section{Kathleen Liddell}

Faculty of Law, University of Cambridge

\section{Roger Clark}

Charles River Laboratories https://orcid.org/0000-0003-4167-6317

\section{Rob Howes}

Cambridge Covid-19 Testing Centre, Discovery Sciences, R\&D, AstraZenenca, Cambridge, UK Michael P. Weekes

University of Cambridge https://orcid.org/0000-0003-3196-5545

\section{Duncan McFarlane}

Institute for Manufacturing, Department of Engineering, University of Cambridge

\section{Patrick H. Maxwell}

University of Cambridge

\section{Nicholas J. Matheson}

University of Cambridge https://orcid.org/0000-0002-3318-1851

\section{Article}

Keywords:

Posted Date: May 20th, 2021

DOI: https://doi.org/10.21203/rs.3.rs-520626/v1 
License: (c) (i) This work is licensed under a Creative Commons Attribution 4.0 International License. Read Full License 
4 Ben Warne ${ }^{1,2,3, *}$, Jessica Enright ${ }^{4}$, Marina Metaxaki ${ }^{1}$, Stewart Fuller ${ }^{1}$, Richard J Samworth ${ }^{5}$,

5 Gillian Weale $^{6}$, Jon Holgate ${ }^{7}$, Vijay Samtani ${ }^{7}$, Craig Brierley ${ }^{8}$, Dinesh Aggarwal ${ }^{1,3,9,10}$, Sarah

6 Hilborne$^{1}$, Mahin Bagheri Kahkeshi ${ }^{1}$, Sarah Berry ${ }^{1}$, Julie A. Douthwaite ${ }^{11}$, Alexandra L.

7 Orton $^{11}$, Rebecca Clarke ${ }^{12}$, Darius Danaei ${ }^{12}$, Rory Dyer ${ }^{12}$, Rob Glew ${ }^{12}$, Oliver Lambson ${ }^{12}$, 8 Aastha Dahal ${ }^{13}$, Ben Margolis ${ }^{13}$, Edna Murphy ${ }^{14}$, Matthew Russell ${ }^{14}$, Vickie S. Braithwaite ${ }^{15,16}$,

9 Kathryn Faulkner ${ }^{15}$, Elizabeth Wright ${ }^{15}$, Afzal Chaudhry ${ }^{1,3}$, Lenette Mactavous ${ }^{3}$, Maharshi

10 Dhada $^{12}$, Ajith Parlikad ${ }^{12}$, University of Cambridge Asymptomatic COVID-19 Screening 11 Programme Consortium ${ }^{\#}$, Jane Greatorex ${ }^{17}$, Ian Leslie ${ }^{7}$, Andy Neely ${ }^{18}$, Steve Rees ${ }^{11}$, Ashley 12 Shaw $^{3}$, Martin Vinnell ${ }^{19}$, Linda Sheridan ${ }^{15}$, Emmeline Watkins ${ }^{15}$, Stephen Baker ${ }^{1,2}$, Ian G. 13 Goodfellow ${ }^{20}$, Paul J. Lehner ${ }^{1,2,3}$, Kathleen Liddell ${ }^{21}$, Roger Clark ${ }^{22}$, Rob Howes ${ }^{11, \dagger}$, Michael P. 14 Weekes ${ }^{1,3,23, \dagger}$, Duncan McFarlane ${ }^{12,24 \dagger}$, Patrick H. Maxwell ${ }^{23,25, \dagger}$, Nicholas J. Matheson ${ }^{1,2,3,26, \dagger *}$

16 1. Department of Medicine, University of Cambridge, UK

17 2. Cambridge Institute of Therapeutic Immunology and Infectious Disease (CITIID), Jeffrey 18 Cheah Biomedical Centre, University of Cambridge, UK

19 3. Cambridge University Hospitals NHS Foundation Trust, UK

20 4. School of Computing Science, University of Glasgow, UK

21 5. Statistical Laboratory, Centre for Mathematical Sciences, University of Cambridge, UK

22 6. COVID-19 Operations Centre, University of Cambridge, UK

23 7. University Information Services, University of Cambridge, UK

24 8. Office of External Affairs and Communications, University of Cambridge, UK

25 9. Public Health England, London, UK.

26 10. Wellcome Sanger Institute, Hinxton, Cambridge, UK. 
11. Biopharma RD, AstraZeneca, Cambridge, UK

12. Institute for Manufacturing, Department of Engineering, University of Cambridge, UK

13. Cambridge Students' Union, Cambridge, UK

14. Office of Intercollegiate Services Ltd., Cambridge, UK

15. Public Health Directorate, Cambridgeshire County Council and Peterborough City Council, UK

16. MRC Epidemiology Unit, University of Cambridge, UK

17. Lucy Cavendish College, University of Cambridge, UK

18. Vice Chancellor's Office, University of Cambridge, UK

19. Occupational Health and Safety Services, University of Cambridge, UK

20. Division of Virology, Department of Pathology, University of Cambridge, UK

21. Centre for Law, Medicine and Life Sciences, Faculty of Law, University of Cambridge, UK

22. Charles River Laboratories, Chesterford Research Park, Saffron Walden, UK

24. St John's College, University of Cambridge, UK

25. School of Clinical Medicine, University of Cambridge, UK

26. NHS Blood and Transplant, Cambridge, UK

\# Full list of consortium members and affiliations is provided in the supplementary materials

$47 \quad$ †Joint senior authors 


\section{Abstract}

53 Transmission of SARS-CoV-2 without symptoms is well described, and may be mitigated by 54 mass testing. Nonetheless, the optimal implementation and quantitative real-world impact of 55 this approach remain unclear. During a period of rising SARS-CoV-2 prevalence, students at 56 the University of Cambridge were enrolled in a voluntary programme of weekly PCR-based 57 asymptomatic screening. Swab pooling by household reduced the total testing capacity

58 required by five-fold, without affecting laboratory workflows or compromising test sensitivity.

59 Participation remained $>75 \%$ throughout the study period. $299 / 671$ (45\%) of students

60 diagnosed with SARS-CoV-2 were either identified or pre-emptively quarantined because of

61 the screening programme. After a negative screening test, the risk of developing COVID-19

62 over the following 7 days was decreased by $51 \%$. Modelling transmission using parameters

63 from our study suggests a reduction in R0 of up to $31 \%$ attributable to weekly screening. We

64 therefore demonstrate the feasibility and efficacy of regular, voluntary mass testing for 65 COVID-19. 
67 Effective control of coronavirus disease 2019 (COVID-19) through a combination of case isolation, household quarantine, and contact tracing/quarantine requires near-complete ascertainment of infectious cases ${ }^{1-3}$. Infection with severe acute respiratory syndrome coronavirus 2 (SARS-CoV-2) in the absence of symptoms is well documented ${ }^{4-6}$, however, and a substantial proportion of onward transmission has been associated with presymptomatic individuals (before they develop symptoms) and/or asymptomatic individuals (who never develop symptoms at all) (w,8 $^{7}$ Strategies focussed on the identification and isolation of symptomatic cases have therefore been ineffective in controlling the COVID19 pandemic.

Mass testing (large-scale screening of populations in the absence of symptoms) has the potential to identify presymptomatic and asymptomatic individuals with SARS-CoV-2 infection. Modelling studies suggest that weekly or twice-weekly screening, together with quarantine of cases and their contacts, will substantially reduce transmission ${ }^{9-11}$. Regular asymptomatic screening has therefore been routinely deployed in specific settings associated with a high risk or high consequence of infection, such as health and social care $^{12-15}$. It has also been trialled in other settings with high risk of transmission such as prisons and professional sport ${ }^{16,17}$. In England, regular mass testing has recently been extended to schools ${ }^{18}$, and offered to all adults in the community ${ }^{19}$. Nonetheless, clear empiric evidence of efficacy is limited, and the approach remains controversial ${ }^{20,21}$.

Multiple outbreaks of COVID-19 have occurred in higher education institutions, often associated with large social gatherings and student accommodation, in spite of infection control measures introduced to prevent viral transmission ${ }^{22-24}$. Amongst university students (typically, healthy young adults), the rate of asymptomatic infection is expected to be higher than in the general population ${ }^{25,26}$. In principle, mass testing is therefore particularly suited to preventing transmission between university students. Controlling transmission in the student 
population also has the potential to reduce viral spread to university staff and members of

93 the local community, who may be at higher risk of developing severe disease ${ }^{27,28}$. Lessons

94 learnt from mass testing in universities should be broadly applicable to asymptomatic screening for COVID-19 in other settings.

Variable levels of information on asymptomatic screening programmes at European and North American universities are available from institutional websites, but outcomes of these programmes have generally not been formally evaluated. Existing reports have focussed on the practicability and acceptability of asymptomatic screening ${ }^{29,30}$ or demonstrated enhanced case ascertainment ${ }^{31-33}$, but provided limited epidemiological metadata or direct evidence for a reduction in transmission on campus or in the community. Despite several modelling studies $^{34,35}$, the quantitative real-world impact of mass testing on SARS-CoV-2 transmission in higher education settings therefore remains unclear.

Regular screening of student populations requires large numbers of tests. One option is to bypass the need for fixed laboratory capacity by utilising community testing with lateral flow tests (LFTs) $)^{36,37}$. Another option is to use laboratory polymerase chain reaction (PCR) tests, but screen individuals in pools. Pooling approaches have the potential to increase greatly the efficiency of resource utilisation where testing capacity is limited. Most studies have relied on combining samples from multiple individuals in the laboratory (sample pooling) ${ }^{38-45}$. This method requires additional sample manipulation, and results in dilution of viral RNA present in individual samples. Alternatively, tests may be pooled at the time swabs (samples) are taken, by combining multiple swabs from individuals in the same sample tube (swab pooling, also known as cohort pooling, or pooling at source $)^{46-48}$. This approach avoids reductions in test sensitivity caused by sample dilution, and disruption of existing laboratory workflows. In this study, we describe the implementation and validation of swab pooling and PCR testing in a UK university, and demonstrate how this approach can be used for weekly asymptomatic screening of student households. At the same time, we present a detailed epidemiological description of the characteristics and spread of COVID-19 within the 
119 university, identifying risk factors for infection and linking secondary attack rates amongst 120 household contacts with the characteristics of index cases. By combining these data, we 121 provide evidence for the efficacy of asymptomatic screening which goes beyond simple 122 numerical case ascertainment. We demonstrate how regular screening is able to identify 123 many students with presymptomatic as well as asymptomatic SARS-CoV-2 infection, 124 quantify the reduction in risk of COVID-19 associated with a negative screening test, and 125 use model-based analysis parameterised from our study to quantify the ability of mass 126 testing to reduce transmission. 


\section{Results}

128

129

130

131

132

133

134

135

136

137

138

139

140

141

142

143

144

145

146

147

148

149

150

151

152

\section{Implementation of asymptomatic screening programme and characteristics of study}

participants

The prevalence of COVID-19 amongst older teenagers and young adults in the UK rose steeply in autumn 2020 , reaching approximately $1.5 \%$ by October $1,2020{ }^{49}$. Against this backdrop, 12,781 students living in University of Cambridge accommodation participated in a weekly asymptomatic COVID-19 screening programme during the autumn term, spanning 9 weeks between October 5, 2020 and December 6, 2020 (Fig. 1). In addition to asymptomatic screening, the University of Cambridge also provided a dedicated, PCRbased testing service for all students and staff with cardinal symptoms of COVID-19 (fever, cough, and/or anosmia/ageusia). Amongst study participants, 1,031 undertook at least 1 of these symptomatic tests during the study period (Fig. 1).

A detailed description of the asymptomatic screening programme is provided in the Methods, and further information is available from the University of Cambridge website

\section{(https://www.cam.ac.uk/coronavirus/stay-safe-cambridge-uni/asymptomatic-covid-19-}

screening-programme). In brief, students were screened using swab pooling and two-step confirmatory PCR testing (Fig. 2). Combined nose and throat swabs were obtained by selfadministration in the students' own accommodation. Swabs from up to 10 students were then immediately pooled in the same tube of viral transport medium. In general, testing pools corresponded with student households. In the event of a positive pooled screening test, students in the pool were instructed to self-isolate, and invited for same-day individual PCR confirmatory testing. Students with positive individual confirmatory tests were treated in the same way as students with positive results from symptomatic testing, including self-isolation, household quarantine and contact tracing. If all individual confirmatory tests were negative, students were released from self-isolation, typically after 1-2 days (depending on whether 1 or 2 rounds of individual tests were required) (Fig. 2). 
University accommodation was divided into 3,094 households (mean 5.0 household

154 members, range 1-20) (Fig. 3a). For pooled sample collection, students were organised into 1552,275 testing pools (mean 6.8 students, range 1-10) (Fig. 3b). To minimise the number of screening tests required, 1,476 (47.7\%) smaller households were combined in merged testing pools. To ensure a maximum of ten swabs per sample tube, $151(4.9 \%)$ larger households were spilt between multiple testing pools. Overall, $65 \%$ of testing pools corresponded exactly with households, $21 \%$ of pools included more than one household, $10 \%$ of pools included a part of one household, and $4 \%$ of pools included students from more than one household and part of one household. Participation was higher by household than by individual student, increasing from $91.3 \%$ (week 1) to $92.9 \%$ (week 9 ) of all households with eligible students (Fig. 3c).

Compared with the overall population of eligible students, the characteristics of participating students were similar (Supplementary Table 1). Undergraduates (particularly first year students and those studying science and technology courses) were somewhat better represented, with small but significant differences in sex, age, ethnicity and UK/international residency. The proportion of students consenting to participate increased over the course of the term, from $75.2 \%$ (week 1) to $81.9 \%$ (week 9) of all eligible students (Fig. $\mathbf{3 d}$ and Supplementary Table 2). Spikes in recruitment were observed after email communications to students providing information about the programme (Extended Data Fig. 1). In theory, the total testing capacity required each week (including both pooled screening and individual confirmatory tests) was expected to vary according to testing pool size and the prevalence of infection amongst screened students (Fig. 3e). In practice, the programme was scaled up in stages over the course of the university term, by increasing the number of students invited to participate in pooled sample collection each week (weeks 1-2, 2 students 177 from each testing pool; weeks 3-7, half the students from each testing pool, alternating week by week; weeks 8-9, all the students from each testing pool, every week) (Fig. 3c,d). At full 
179

180

181

182

183

184

185

186

187

188

189

190

191

192

193

194

195

196

197

198

199

200

201

202

203

204

scale (weeks 8-9), utilisation of testing capacity was almost 5x more efficient than screening with individual tests alone (Fig. 3e and Supplementary Table 2).

\section{Clinical performance of pooled screening tests}

In total, 16,945 pooled screening tests were analysed between October 5, 2020 and December 6, 2020 (weeks 1-9), of which 252 were positive (Supplementary Table 3). $205 / 252(81.3 \%)$ of these tests were followed by at least one positive individual confirmatory test (Fig. 4a and Supplementary Table 3), increasing to 183/189 (96.8\%) for pooled screening tests with cycle threshold (CT) values $\leq 36$. Amongst all pooled screening tests, approximately $1 / 360$ were characterised pragmatically as "operational false positives" (a positive pooled screening test, followed by negative individual confirmatory tests from all students in the testing pool). Because pooled screening and individual confirmatory tests were conducted on separate samples obtained on sequential days, these operational false positives likely reflect some true negative individual confirmatory tests from students with declining viral loads, false negative individual confirmatory tests from students with borderline viral loads, as well as technical false positive screening test results. Amongst positive pooled screening tests, the proportion of operational false positives was lowest in weeks $2(1 / 28,3.6 \%), 3(2 / 38,5.3 \%)$ and $6(8 / 67,11.9 \%)$ when the prevalence of infection amongst screened students was highest. Conversely, the fraction characterised as false positives was highest in weeks $8-9(14 / 17,82.4 \%)$, when the prevalence of infection amongst screened students was lowest (Supplementary Table 3).

Because students contributing swabs to negative pooled screening tests did not undergo paired individual tests, it was not possible to calculate an operational false negative rate for pooled screening tests. Nonetheless, we did not observe any loss in analytical sensitivity for pooled samples containing $\geq 5$ swabs tested using spike-in standards (Extended data Fig. 2), and the limit of detection of the assay $\leq 125$ SARS-CoV-2 digital copies $/ \mathrm{mL}$ viral transport 
media remained well within the UK Medicines and Healthcare Products Regulatory Agency (MHRA) performance specification for laboratory-based SARS-CoV-2 PCR tests (Supplementary Table 4) ${ }^{50}$. There was a good correlation between CT values from pooled screening tests and paired individual confirmatory tests (Fig. 4b), and no differences in the distributions of CT values were observed between paired tests stratified by number of swabs (Fig 4c). Taken together, and in agreement with previous studies ${ }^{46-48}$, these data strongly argue that swab pooling does not result in any significant loss of PCR test sensitivity.

\section{Characteristics of students with SARS-CoV-2 infection}

Over the entire term, $5.2 \%(671 / 12,781)$ of participating students were diagnosed with SARS-CoV-2 infection across all testing routes (Supplementary Table 3). After an initial increase in week 2, the incidence of infection fell gradually during weeks 3-5 (Fig. 5a). A second increase in week 6 followed Halloween and the announcement of the second UK national lockdown, with a dramatic decline in cases over weeks 7-9 with lockdown measures in place. Compared with the overall population of participating students, students diagnosed with SARS-CoV-2 were more likely to be male, of white ethnicity and resident in the UK out of term time, according to both single variable and multivariable models (Supplementary Table 5, 6). There were also significant associations with the first year of study at the university, studying arts and humanities subjects at undergraduate level and living in a larger household. Other determinants of transmission within the university, and the impact of lockdown measures, were analysed using genomic epidemiology and contact tracing data in a separate report ${ }^{51}$.

In total, 39.2\% (256/671) of all participating students with SARS-CoV-2 infection were identified by the asymptomatic screening programme (range $0-75 \%$, week by week) (Supplementary Table 3). To determine how many of these students experienced symptoms at any time during their infection, we conducted a retrospective telephone survey, 
and obtained 140/256 (54.7\%) responses. Amongst respondents, 68/140 (48.6\%) reported

232 cardinal symptoms of COVID-19 (fever, cough and/or anosmia/ageusia) at some point in 233 their infection (Extended Data Fig. 3a,b). The distribution of time intervals between positive

234 pooled screening tests and symptom onset for these students implies a variable presymptomatic infectious period, with a mean of 3.6 days (Extended Data Fig. 3c). This is consistent with estimates from other populations, but slightly longer than most (typically 1-4 days) $)^{52}$. This may reflect methodological differences, or the particular demographic characteristics of our study population.

Of the respondents to the survey, $29 / 140(20.7 \%)$ remained entirely asymptomatic. An additional $43 / 140(30.7 \%)$ students detected by the asymptomatic screening programme reported minor symptoms, excluding fever, cough and anosmia/ageusia. Since these students did not meet criteria for self-isolation or symptomatic testing, they were classified pragmatically with asymptomatic students.

\section{Viral RNA shedding and transmission of SARS-CoV-2 in student households}

Compared with students with SARS-CoV-2 infection identified by symptomatic testing, CT values from students identified by asymptomatic screening were somewhat higher (mean CT values 24.8 vs $27.2, P<0.0001 ; 59 \%$ vs $42 \%$ CT values $<25, P<0.0001)$, suggesting lower viral loads (Extended Data Fig. 4a and Supplementary Table 5). We therefore stratified CT values from students sampled by our telephone survey, according to the presence

251 (presymptomatic students) or absence (asymptomatic students) of cardinal symptoms of 252 COVID-19 at some point in their infection. CT values were lower for presymptomatic students (mean CT value 25.5; $53 \%$ CT values $<25$ ), similar to students identified by symptomatic testing (Extended Data Fig. 4b and Supplementary Table 5). These results are consistent with the known peak in RNA shedding early in SARS-CoV-2 infection, typically on or just before symptom onset, and coinciding with symptomatic testing or 
identification of presymptomatic students ${ }^{53,54}$. Conversely, within the limit of the screening

258 interval, asymptomatic students may be sampled at any point in their infection.

259 Next, we assessed the number of secondary infections amongst household contacts of 260 students with SARS-CoV-2 infection (index cases). Compared with index cases identified by 261 symptomatic testing, the secondary attack rate for index cases identified by asymptomatic screening was lower (6.9\% vs $12.0 \%$ of household contacts, $P=0.0003)$ (Extended Data Fig. 5a). Similar to CT values, we therefore stratified secondary attack rates for index cases sampled by our telephone survey, according to the presence (presymptomatic students) or absence (asymptomatic students) of cardinal symptoms of COVID-19 at some point in their infection. Remarkably, the secondary attack rate was much higher for presymptomatic students $(15.2 \%)$, similar to index cases identified by symptomatic testing (Extended Data Fig. 5b). Conversely, the secondary attack rate for asymptomatic students was much lower (2.3\%), consistent with the differences observed in CT values, and suggesting a lower infectiousness (transmission potential) of students who never develop symptoms (relative risk 0.17 compared with symptomatic students, $95 \%$ C.I. $0.08-0.38)$. This is consistent with estimates from other populations ${ }^{8}$. Whilst some infections amongst household contacts may have been acquired outside the home, that would tend to bias towards the null (smaller relative risk reduction for asymptomatic students). Infectious virus is more readily recovered in the laboratory from patient samples with low CT values (higher viral loads), and individuals with low CT values are therefore presumed to be more infectious ${ }^{55}$. To test this hypothesis, we examined the relationship between the secondary attack rate amongst household contacts and the CT value of the index case. As predicted, there was an inverse correlation between the CT value and the secondary attack rate (Extended Data Fig. 5c). Nonetheless, the magnitude of the effect was relatively modest, ranging from $10.3 \%$ (CT<20, highest viral loads) to $5.5 \%$ (CT>30, lowest viral loads) (Extended Data Fig. 5d). 
285 Combining our data on case ascertainment with the results of our telephone survey (and assuming that sampling was random), we estimate that $20 \%$ (95\% C.I. $16.4-22.9 \%)$ of all students with confirmed COVID-19 were asymptomatic (no cardinal symptoms) (Fig. 5b). Of the remaining students with confirmed COVID-19 who developed symptomatic disease, $23 \%$ (95\% C.I. 19.0-27.1\%), were detected by the asymptomatic screening programme. A further $8 \%$ of these students were detected by symptomatic testing, but were already quarantined at the time of symptom onset because of detection of a household contact by the asymptomatic screening programme. Taken together, the asymptomatic screening programme may therefore have contributed to the interruption of onward transmission from $45 \%$ of all students with confirmed SARS-CoV-2 infection.

To measure directly the efficacy of asymptomatic screening for the detection of students with presymptomatic infection using an orthogonal approach, we focussed on weeks 3-7 of the programme. During this period, half the students from each testing pool were screened each week, with a screening interval of 14 days for individual participating students. The risk of a positive symptomatic test was smallest immediately following a negative screening test (Fig. 6a), and the likelihood of a positive symptomatic test 1-7 days after a negative screening test was reduced by approximately $51 \%$ (52 vs 106 students) compared with days $8-14$. This reduction corresponds to the successful detection of presymptomatic cases. It further suggests that weekly asymptomatic screening will detect approximately half of all students who ultimately develop cardinal symptoms of COVID-19 whilst still presymptomatic, in agreement with the presymptomatic infectious period observed in our study (mean 3.6 days).

Finally, to quantify the potential of different screening strategies to reduce SARS-CoV-2 transmission, we parameterised a discrete-timestep forward-simulation SEAIR network model using key real-world metadata derived from our study (Supplementary Table 8).

309 These metadata included: distribution of household sizes (Fig. 3a); within and between 310 household secondary attack rates (Extended Data Fig. 5); proportion of asymptomatic 
311 infections (Fig. 5b); and duration of presymptomatic infectious period (Extended Data Fig.

312 3c). In all simulations, we assumed that symptomatic individuals self-isolate with their

313 households from the day of symptom onset. We projected new cases over time (Fig. $\mathbf{6 b}, \mathbf{c}$ ),

314 and compared the effect of each screening strategies on the basic reproduction number (R0)

315 and the number of students quarantined (Fig. $6 \mathbf{c}, \mathbf{d})$.

316 Weekly screening of all students (as implemented during weeks 8-9 of the programme)

317 resulted in a $31 \%$ reduction in $\mathrm{R} 0$ from a median of $1.78(95 \% \mathrm{Cls} 1.37-2.23)$ to a median of

$3181.22(95 \%$ Cls $0.82-1.62)$, with screening of half the students from each testing pool each

319 week (as implemented during weeks 3-7 of the programme) intermediate in effect (median

$320 \mathrm{R} 01.45,95 \% \mathrm{Cls}-1.02-1.88)$. Similar effects on transmission were observed in all

321 sensitivity analyses (Extended Data Fig. 6), and the number of students quarantined was

322 reduced, rather than increased, by more frequent screening (Fig. 6c,d). These simulations

323 therefore support the efficacy of the asymptomatic screening programme for the control

324 SARS-CoV-2 transmission in the university. 


\section{Discussion}

326 To our knowledge, this is the most comprehensive epidemiological evaluation to date of 327 regular, large-scale asymptomatic screening for COVID-19 in a university or any other defined residential setting. The duration of SARS-CoV-2 RNA shedding from the upper respiratory tract is typically $2-3$ weeks ${ }^{56,57}$. Even allowing for the possibility of false negative

330 PCR tests ${ }^{58,59}$, the combination of regular asymptomatic screening with readily accessible 331 symptomatic testing is therefore likely to have detected almost all cases of SARS-CoV-2 infection in our study population, which could then be mapped to the known household structure.

Amongst all students with confirmed SARS-CoV-2 infection, the proportion of asymptomatic students $(20 \%)$ was comparable with overall estimates from other studies ${ }^{7,8}$, but lower than predicted given the age range of study participants. This may reflect the retrospective ascertainment of symptoms, including subjective loss or change to the sense of smell or taste (which may be more frequent in young adults, and mild disease) ${ }^{60,61}$. Conversely, the proportion of infected individuals who ultimately developed symptoms identified by asymptomatic screening (25\%) was greater than predicted, given that weekly screening of all participating students was only achieved by week 8 . Nonetheless, this proportion is in keeping with the observed reduction in likelihood of symptomatic disease in the week after a negative screening test $(51 \%)$, as well as the observed presymptomatic infectious period

\section{4 (3.6 days).}

345 Consistent with the known peak in SARS-CoV-2 viral load early in infection (on or just before 346 symptom onset), CT values in presymptomatic students were low, and comparable to CT 347 values in symptomatic students ${ }^{53}$. In agreement with this, the observed secondary attack 348 rate amongst household contacts was significantly greater for presymptomatic and symptomatic index cases, than index cases who never developed symptoms. Taken together, these observations support our classification of presymptomatic and asymptomatic 
students, and imply that the infectiousness of students who never develop symptoms is

352 significantly reduced. They are consistent with data from another recent study ${ }^{62}$, and suggest

353 that screening programmes should be designed to target individuals with presymptomatic

354 infection, for example by maximising testing frequency.

355 Quantifying the efficacy of our screening programme by comparison with different universities is not possible, because of the variable and multi-faceted infection prevention and control measures deployed by different institutions. Nonetheless, we are able to present direct evidence for the efficacy of regular asymptomatic screening: first, enhanced case ascertainment, including students with presymptomatic as well as asymptomatic infection

360 (39\% of all cases); second, the pre-emptive quarantine of students with symptomatic

361 COVID-19 infection, because of a positive household contact detected by the screening programme ( $7 \%$ of all cases); and third, the reduction in risk of symptomatic COVID-19 in the period following a negative screening test result (corresponding to the successful detection of presymptomatic cases). This direct evidence is strongly supported by indirect evidence from our model-based analysis, parameterised empirically using data from our study. Genomic epidemiology and contact tracing data from our student cohort are presented in a separate report, and confirm the impact of asymptomatic screening and other infection control policies in limiting introductions of SARS-CoV-2 to the university, containing viral spread within student accommodation, and restricting transmission between the university and the surrounding community ${ }^{51}$.

371 Our screening programme was deployed within 8 weeks of inception without pre-existing clinical, information technology or logistics infrastructure. Swab pooling from up to 10

373 students is compatible with standard nasopharyngeal swabs and sample tubes, and ensures

374 that samples can be analysed without significant changes to laboratory workflow. Taking into 375 account variation in household size and the requirement for individual confirmatory testing, 376 this approach increases effective PCR testing capacity roughly three- to five-fold for SARS-

377 CoV-2 prevalences up to $2 \%$. It is particularly well-suited to residential settings, where 
378 testing pools correspond with units of household quarantine e.g. universities, long-term care

379 facilities, prisons, workers housed in dormitories, or private households. Nonetheless, it may 380 also be applied in other settings where individuals are grouped together, such as schools or 381 workplaces ${ }^{63}$.

382 Our data suggest that voluntary participation and the use of nose and throat swabs (rather 383 than less invasive sampling, such as saliva) are not per se barriers to widespread uptake amongst students, and likely other populations. Other factors which may have enhanced participation in our programme include: ease of access and convenience of home testing, a proactive communication strategy, engagement with student representatives, the collegiate university structure, the use of household-based pooled screening, and careful attention to ethical and legal issues (e.g. personal data governance). Many of the approaches taken in the development, operation and evaluation of our programme have been supported by a recent ethical review of asymptomatic COVID-19 screening programmes in higher education settings ${ }^{64}$.

Criticisms of mass testing for COVID-19 typically have typically focussed on the potential for false positives (leading to unnecessary periods of quarantine) and false negatives (leading to missed cases) ${ }^{20,21}$. Our two-step testing strategy (including individual confirmatory PCR tests) mitigates the chance of false positive tests leading to prolonged periods of unnecessary individual or household quarantine. Moreover, swab pooling effectively increases the prevalence of true positive samples. In theory, the high sensitivity of PCR tests could result in the detection of individuals with low levels of viral RNA late in their course of infection, even when they are no longer infectious ${ }^{65}$. Whilst this is a valid concern for one-off mass testing, it is not relevant for programmes based on regular, frequent screening.

401 Conversely, the high sensitivity of PCR tests ensures that the risk of false negatives is 402 minimised. This may be particularly important for the detection of breakthrough infections 403 following vaccination, when viral loads may be reduced ${ }^{66}$. At the same time, PCR testing 
404 facilitates the reliable reporting of cases from laboratories to public health systems, and the

405 provision of samples for viral sequencing to support surveillance and transmission studies ${ }^{51}$.

406 LFTs offer a relatively cheap alternative to laboratory-based testing, typically return results in 407 only 30 minutes, and can be deployed rapidly at scale with minimal fixed infrastructure. They 408 have therefore been used in the UK and elsewhere for regular and one-off mass testing in 409 different settings ${ }^{19,67}$. The false positive rate of these tests is comparable to the operational 410 false positive rate observed in our programme, and may be mitigated by confirmatory PCR 411 testing ${ }^{36}$. When LFTs are conducted by trained staff and volunteers, however, sensitivity for 412 SARS-CoV-2 detection is roughly $50 \%$ compared with PCR tests ${ }^{58,68,69}$, and their 413 performance when used for home testing is not well documented. In general, the false 414 negative rate is lower for individuals with high viral loads, who are likely to be most 415 infectious ${ }^{65}$. Nonetheless, the difference in secondary attack rates amongst household 416 contacts of index cases with high or low CT values observed in our study suggests that this 417 gradient in infectiousness may be modest, consistent with variability in serial sampling ${ }^{53,70}$, 418 laboratory data on recovery of infectious virus ${ }^{55}$, and analysis of contact tracing data ${ }^{71}$. 419 Notwithstanding the rapid turnaround of LFTs, and asymmetric profile of SARS-CoV-2 viral 420 shedding, it is therefore likely that adherence to twice weekly mass testing using home LFTs 421 will be required to match or even exceed the benefits of weekly PCR-based screening ${ }^{54}$.

422 The development of SARS-CoV-2 variants with mutations enhancing transmissibility and 423 enabling at least partial escape from vaccine-induced and natural immunity ${ }^{72-74}$, together 424 with the limited availability of vaccination on a global scale ${ }^{75}$, suggest that non425 pharmaceutical interventions to control the transmission of SARS-CoV-2 will be required for 426 some time yet. Our study was conducted in the UK before the widespread circulation of 427 B.1.1.7 lineage viruses ${ }^{76}$. Although the relative efficacy of mass testing will likely be retained 428 for different lineages, the precise impact may be subject to changes in transmission 429 dynamics associated with different variants, such as the presymptomatic infectious period. 430 Compared with social distancing and lockdown measures, the social and economic 
431 consequences of mass testing are much less severe. Taken together, our data strongly 432 support the role of regular asymptomatic screening as an effective means to control SARS433 CoV-2 transmission, in universities as well as other settings. 


\section{Methods}

\section{Ethics statement}

436 Ethical approval for this study was granted by the University of Cambridge Human Biology

437 Research Ethics Committee (HBREC.2020.35). Participation was voluntary, with no coercion

438 or incentives. Informed consent was obtained from all participants, who were free to

439 withdraw from the programme at any time. All data was processed fairly, lawfully and

440 transparently, in accordance with the EU General Data Protection Regulation (GDPR)

$4412016 / 679$ and the UK Data Protection Act 2018. The student information sheet, privacy

442 notice, consent form, protocols for sample collection, additional information and answers to

443 frequently asked questions are available on the programme website

444 (https://www.cam.ac.uk/coronavirus/stay-safe-cambridge-uni/asymptomatic-covid-19-

445 screening-programme).

447 Study design and participants

448 The Asymptomatic COVID-19 Screening Programme is a weekly screening programme for

449 SARS-CoV-2 infection for students at the University of Cambridge. During autumn

450 (Michaelmas) term 2020, screening took place over 9 weeks from October 5, 2020 to

451 December 6, 2020. Students were screened using swab pooling and two-step confirmatory

452 PCR testing (Fig. 1). Additional tests were provided by the university for all students and

453 staff with symptoms of possible COVID-19.

454 The University of Cambridge comprises 31 colleges. All undergraduate and postgraduate 455 students resident in university (college) accommodation were eligible for participation in the 456 programme, along with resident students of the Cambridge Theological Federation (weeks 457 1-9). To accord with UK government guidance on student testing prior to the Christmas 458 vacation 2020 , eligibility was extended to include students resident in private 
accommodation on November 30, 2020 (week 9). Results from these students will be

460 described in a separate report.

461 Eligible students were invited to participate by email. The programme was initially

462 announced to students on September 9, 2020 by email communication. Formal email

463 invitations were sent to all eligible students on September 26, 2020 and weekly thereafter.

464 Each email contained links to the programme website, which included the consent form, 465 student information sheet and privacy notice. Additional communications were sent via email

466 from individual colleges and promoted on social media by student representatives.

467 The screening programme was introduced in phases, to ensure that all aspects of the system operated effectively and had capacity to manage greater numbers. Initially, two students from each testing pool were randomly selected to contribute swabs for pooled sample collection each week (weeks 1-2). Then, half of the students in each testing pool

471 were asked to contribute swabs each week (weeks 3-7, ensuring every student was tested 472 every two weeks). Then, all students in each testing pool were asked to contribute swabs 473 each week (weeks 8-9, ensuring every student was tested weekly).

474 At any point in the term, students were excluded from pooled sample collection if: 1) they 475 had tested positive for SARS-CoV-2 within the preceding 8 weeks; 2) they had symptoms of 476 possible COVID-19 or were awaiting an individual symptomatic test result; 3) they were self477 isolating for any other reason, for example due to contact with a confirmed or suspected 478 COVID-19 case or international travel from a high prevalence area; or 4) for weeks 1-7 only, 479 they had not been selected to participate (see above).

\section{Asymptomatic screening programme}

482 Students living in university accommodation with high-contact shared facilities such as a 483 bathroom, toilet or kitchen area are considered to share the same household. For the purposes of screening, some households were split or merged with neighbouring 
households to ensure testing pools of 6-10 students. Household and testing pool membership were determined by each college prior to the start of term (based on guidance provided by the university and the screening programme) and recorded in a central database that could be updated at any time. This database provided a single reference point used for test kit production and result notification (see below).

Bespoke screening kits were prepared within the university. Each kit was labelled with the names of students belonging to a particular testing pool, and contained packaging and cleaning materials, a number of nasopharyngeal flocked swabs (A04-96000P, Trafalgar Scientific, or MSC-96000, Sterilab) corresponding to the number of students within that pool, and one tube of viral transport medium (HiViral Transport Medium, Trafalgar Scientific) labelled only with a pseudonymised code associated with that testing pool. Screening kits were delivered to each college weekly for distribution to testing pools. Each college was assigned a testing day, with all students from that college invited to participate on the same day. Drop-off points were allocated in every college, with same-day sample collection and delivery to the testing facility ensuring the availability of results within 24 hours. Screening took place Mon-Thurs, with individual confirmatory tests (see below) Tues-Fri.

501 A detailed swabbing protocol is available from the programme website. In brief, combined 502 nose (anterior nasal) and throat swabs were obtained by self-administration in the students' 503 own accommodation. Swabs from all students in a testing pool were immediately pooled in 504 the same tube of viral transport medium. Each tube had capacity for up to 10 swabs, which therefore determined the maximum number of students in a testing pool. To confirm the number of students participating each week, one member of each testing pool was asked to complete a simple electronic form at the time of pooled sample collection, indicating the number of swabs present in the sample tube ("swab count").

509 All samples were tested by PCR in the established University of Cambridge/AstraZeneca

510 Cambridge COVID-19 Testing Centre (see below). Pseudonymised results were transferred 511 to the secure data hosting service (SDHS) provided by the University of Cambridge (School 
512 of Clinical Medicine), where they were associated with members of each testing pool.

513 Results were typically communicated to students by text message, and shared with colleges

514 and the university COVID-19 Operations Centre via the SDHS. In addition, results of

515 individual confirmatory tests (see below) were reported to Public Health England using the

516 Second Generation Surveillance System (SGSS).

517 Students contributing swabs to positive pooled screening tests were instructed to self-

518 isolate, and either invited for same-day individual confirmatory tests at a University of

519 Cambridge/Cambridge University Hospitals NHS Foundation Trust (CUHNFT) testing facility

520 in the city centre (weeks 1-6, see below) or provided with a test kit for individual self-

521 swabbing in their own accommodation (weeks 7-9). Students with positive individual

522 confirmatory tests were treated in the same manner as students with positive results from

523 existing University of Cambridge or NHS pathways for symptomatic testing. In all cases,

524 isolation of cases and contacts was in accordance with UK national guidance, instructions

525 from NHS Test and Trace and the local public health teams.

526 In brief, cases were required to self-isolate for 10 days from their date of symptom onset (or

527 test date, if asymptomatic). Households of confirmed cases were quarantined for 14 days. If

528 a positive student could not be identified by individual testing, the CT value of the positive

529 pooled screening test was reviewed (Fig. 2): if $>36$, the pooled screening test was

530 considered to be an operational false positive; if $\leq 36$, a further round of individual tests was

531 performed, to minimise the risk of a false negative individual test result. If all individual

532 confirmatory tests were negative, students were released from self-isolation/household

533 quarantine. This approach is concordant with NHS guidelines for individual PCR tests which

534 are positive at the limit of detection.

535 
537 In addition to asymptomatic screening, the University of Cambridge (in partnership with

538 CUHNFT) also provided a dedicated testing service for all students and staff with cardinal

539 symptoms of COVID-19 (fever, cough, and/or anosmia/ageusia). Combined nose and throat

540 swabs were obtained by self-administration at a university testing facility in the city centre,

541 Mon-Fri during the study period. Alternative testing via NHS pathways was also available to

542 all students.

543 Individual results from the university's symptomatic testing pathway were reported via the 544 electronic medical record at CUHNFT (Epic Systems, Verona, WI, USA). Ascertainment of

545 students testing positive by NHS pathways was derived from self-reporting to the university

546 COVID-19 Operations Centre, completion of the university's COVID-19 monitoring form, or

547 information provided by the Local Authority or Public Health England as part of investigations

548 into transmission networks during the study period.

\section{Other control measures}

551 A detailed description of additional COVID-19 reduction measures implemented by the

552 University of Cambridge, including social distancing and the use of face coverings, is 553 provided in a separate report ${ }^{51}$.

Telephone survey

556 To investigate the presence, nature and timing of symptoms experienced by students with 557 SARS-CoV-2 infection identified by asymptomatic screening, we conducted a retrospective telephone survey of all students with positive individual confirmatory tests. Students were contacted between November 24, 2020 and December 17, 2020 by clinical research nurses or members of the university COVID-19 Operations Centre (contact tracing team), and 
561 assessed using an adapted pro forma employed for screening symptomatic staff members at

562 CUHNFT. In brief, students were asked if they developed cardinal symptoms of COVID-19

563 (fever, cough, and/or anosmia/ageusia) or other minor symptoms (such as sore throat, 564 headache, myalgia, fatigue, breathlessness or gastrointestinal symptoms) before their 565 positive test result, or during their period of self-isolation.

566 To estimate the proportions of all students identified by asymptomatic screening who experienced symptoms, the sample of students responding to the telephone survey was assumed to be representative and independent of the manifestation of symptoms. Binomial confidence intervals were calculated using the Clopper-Pearson interval.

570

571 Laboratory procedures and validation of swab pooling

572 All samples were tested by PCR in the University of Cambridge/AstraZeneca COVID-19

573 Testing Centre (Anne McLaren Building, Cambridge Biomedical Campus), part of the UK

574 Lighthouse Labs Network, in accordance with existing procedures for clinical samples. In 575 brief, RNA was extracted from heat-inactivated samples using the Beckman RNA Advance 576 Viral Kit, then tested by RT-qPCR for the presence of the orf1ab gene of SARS-CoV-2 using 577 the Genesig Real Time PCR COVID-19 High Throughput (HT) Assay Kit.

578 To investigate the impact of swab pooling on SARS-CoV-2 detection, swabs and residual 579 viral transport medium (VTM) from negative samples were used to reconstitute mock 580 samples containing known concentrations of SARS-CoV-2, in the presence of either a single 581 swab (individual samples) or 5-8 swabs (pooled samples). VTM volumes were normalised to $5821 \mathrm{~mL}$, spiked with serial dilutions of SARS CoV-2 (250,000 to 125 digital copies $/ \mathrm{mL})$ using 583 the Qnostics SARS Cov-2 Analytical Q Panel (whole, inactivated SARS-CoV-2 virus), then 584 incubated for $1.5 \mathrm{~h}$ and tested using existing procedures for clinical samples. 
585 Where indicated, distributions of CT values of pooled or individual tests were compared

586 using paired or unpaired 2 sample 2-tailed $t$-tests (comparison of 2 distributions) or ordinary

587 one-way ANOVA (comparison of 3 or more distributions), as specified in the figure legends.

588

589 Other statistical analyses

590 All pooled screening, individual confirmatory and symptomatic test results were linked with

591 participating students, households and testing pools in a single pseudonymised database.

592 Participation rates were estimated by comparing the number of samples received and

593 reported by the testing facility with the mean number of students participating per testing

594 pool, as determined by "swab counts" (see above). Student characteristics associated with

595 programme participation were assessed using unpaired 2 sample 2-tailed $t$-tests (continuous

596 variables) or chi-square tests (categorical variables), as specified in the table legend.

597 To evaluate the secondary attack rate amongst household contacts, we first identified all

598 index cases (the first case diagnosed in each household). For each index case, we then

599 compared the number of household contacts testing positive within 14 days of the index,

600 compared with the total number of household contacts. Results were stratified according to

601 the characteristics of the index case (testing route, symptoms and CT values of their

602 individual sample). Where multiple index cases were identified in the same household on the

603 same day, they were either reported as a separate "mixed" category or the household was

604 excluded from analysis. Secondary attack rates were compared using Fisher's exact test.

605 Confidence intervals for the relative risk estimates were calculated using the Koopman

606 asymptotic score method. To assess the relationship between the CT value of each index

607 case and the probability of household contacts testing positive within 14 days, fitted

608 probabilities were obtained from a logistic regression model.

609 To calculate the presymptomatic infectious period in our study population, a positive PCR

610 test was taken as proxy for infectiousness. Data were analysed from 68 students identified 
611 by asymptomatic screening, who reported cardinal symptoms of COVID-19 at some time

612 during their infection (presymptomatic students). Provided the screening interval is longer

613 than the presymptomatic infectious period, presymptomatic students will be sampled at

614 random during this period, and (on average) halfway through. We therefore calculated the

615 mean presymptomatic infectious period as twice the mean observed time interval between a

616 positive pooled screening test and symptom onset.

617 To estimate the number of students with presymptomatic SARS-CoV-2 infection detected by

618 asymptomatic screening, we reasoned that successful identification of these cases should

619 correspondingly reduce the number of students identified by symptomatic testing in the

620 period after a negative screening test, in accordance with the duration of the presymptomatic

621 infectious period. We therefore analysed data from 158 students with SARS-CoV-2 infection

622 identified by symptomatic testing during weeks 3-7 of the programme. Over this period, half

623 the students from each testing pool were screened each week, corresponding to a screening

624 interval of 14 days for individual participating students. For each student, we assessed the

625 time interval between their positive symptomatic test and their most recent negative

626 screening test. We then compared the number of students testing positive 1-7 days or 8-14

627 days after a negative screening test. We selected these periods because: 1) we observed a

628 mean presymptomatic infectious period $\leq 7$ days in most students (mean 3.6 days); 2) we

629 wished to estimate the proportion of students who ultimately develop cardinal symptoms of

630 COVID-19 likely to be detected whilst still presymptomatic in the presence of weekly

631 asymptomatic screening (that is, every 7 days); and 3) the screening interval of 14 days sets

632 an upper bound.

633 To predict the total number of pooled screening and individual confirmatory tests (testing

634 capacity) required to screen every 1,000 participating students in the presence of different

635 testing pool sizes and prevalences of SARS-CoV-2 infection, we assumed that: 1) 1/360

636 pooled screening tests are false positives (equal to the observed rate of operational false

637 positives); 2) test sensitivity is $100 \%$; 3 ) testing pool sizes are homogenous; and 4) positive 
students are evenly distributed throughout the population screened. For each week of

639 screening, predicted testing capacity was compared with the observed mean testing pool

640 size (swab count) and numbers of pooled screening and individual confirmatory tests

641 conducted.

642 Associations between testing positive for SARS-CoV-2 (by any testing route at any time

643 during the study) and individual risk factors were examined by logistic regression and

644 expressed as odds ratios with $95 \%$ confidence intervals and associated $P$ values. All

645 variables were treated as categorical, with the exception of household size, which was

646 treated as continuous.

647 A multivariable logistic regression analysis was fitted with six covariates, namely gender,

648 ethnicity, residence (out of term time), year group, course and household size. All variables

649 were significant, so were retained in the final model. Pairwise interaction terms were also

650 considered, but did not lead to an improved model. To assess the goodness-of-fit, we

651 applied two tests: the classical Hosmer-Lemeshow test ${ }^{77}$ and the more recent Generalised

652 Residual Prediction (GRP) test ${ }^{78}$. The $P$ value from the Hosmer-Lemeshow test was 0.44 ,

653 while the GRP test $P$ value was 0.002 . While the latter suggests that there is potentially

654 some scope for improved predictions using nonparametric techniques such as random

655 forests, the much simpler logistic regression model has great advantages in terms of

656 interpretability and was therefore our preferred model.

657 Data were initially compiled in Azure (Microsoft) and Excel 2013 (Microsoft) and analysed in

658 STATA 14.2 (College Station, TX, USA) and GraphPad Prism version 9.0.0 (GraphPad

659 Software, CA, USA). Single variable and multivariable analyses were performed in R 4.0.3

660 (R Foundation for Statistical Computing, Vienna, Austria) using the glm command. The

661 Hosmer-Lemeshow test is available in the package 'generalhoslem', while the Generalised

662 Residual Prediction test is available in the package 'GRPtests'.

663 
665 To further evaluate the impact of different testing strategies on SARS-CoV-2 cases and

666 numbers of students in isolation over the term, we developed a model parameterised

667 primarily from findings in our study (Supplementary Table 8). This is based on an existing

668 model specifically created to provide insights into the impact of SARS-CoV-2 in UK higher

669 education settings, and developed by a working group from multiple institutions following

670 meetings as part of the Isaac Newton Institute Infectious Dynamics of Pandemics Research

671 Programme ${ }^{79}$. It is an adaptation of the layered network model of student contacts, with one

672 layer for household contacts and another for non-household contacts, and is ultimately

673 based on code produced by Sara Jakubiak available at

674 https://github.com/SaraJakubiak/covid19-caribbean-educational-model. The code used for

675 this manuscript can be found at: https://github.com/magicicada/covid19-caribbean-

676 educational-model/tree/manuscript cambs testing scheme.

677 Students are allocated to households according to a specified distribution of household sizes, and allocated out-of-household contacts in groups according to another specified distribution of activity groups intended to simulate group teaching and social activities. Both

680 household and non-household contacts are produced in groups, and all individuals within the 681 group are pairwise in contact within the network. The disease model is a stochastic SEAIR 682 simple compartmental model in which infectious (I compartment) individuals may be 683 symptomatic or asymptomatic, and presymptomatic (A compartment) individuals are also 684 infectious. Compartments for isolating individuals are also included. Movement between model compartments is by fixed daily probability for each individual. No severe illness or 686 hospitalisation are modelled. For the main results, 2500 simulations were run for each 687 testing regime: 50 different network instantiations with 50 separate simulations on each.

688 We model several asymptomatic testing regimes structured at the household level; in all 689 testing regimes symptomatic individuals are assumed to immediately seek testing. When a 690 positive case is detected, the entire household of the case isolates, as well as a parameter- 

specified proportion of the test-positive's out-of-household contacts. When a household is

692 isolating, infection can occur within the household, but not outside of the household.

693 While a full analysis of the sensitivity of this model to all parameters is not feasible here, 694 given the large number of variables, we report a basic univariate sensitivity analysis on parameters: 1) to which we judge the model likely to be most sensitive; 2) about which we are most uncertain; and 3) for which the structure of the model allows us to straightforwardly adjust. For each of these, we show results of a model run with the parameter decreased by $15 \%$ and increased by $15 \%$. For each parameter setting and testing regime combination, we ran a total of 600 model runs: 20 different network instantiations with 30 separate simulations on each.

While a multivariate analysis would add certainty, this would require a very large number of additional experiments and is beyond the scope of this work. We caution that a number of assumptions that are not straightforwardly adjusted within this model framework are also likely to have an important quantitative (though not qualitative) impact on the outcome of the model, in particular assumptions about perfect adherence to testing and isolation procedures. Evaluating the impact of varying adherence is beyond the scope of this particular investigation.

\section{Role of the funding source}

710 The programme is primarily funded, overseen and implemented by the University of

711 Cambridge, its associated Colleges and the Cambridge Theological Federation. The

712 Cambridge COVID-19 testing facility represents a collaboration between the University of

713 Cambridge, AstraZeneca and Charles River Laboratories, funded by the Department of

714 Health and Social Care. AstraZeneca and Charles River Laboratories reviewed the data

715 from the study and the final manuscript before submission, but the academic authors retained editorial control. Other funders had no role in the study design, data collection, data 
717 analysis, data interpretation, or writing of the report. All authors had full access to all the data

718 in the study and had final responsibility for the decision to submit for publication.

719

720 Data availability

721 Weekly reports are available from the programme website

722 (https://www.cam.ac.uk/coronavirus/stay-safe-cambridge-uni/asymptomatic-covid-19-

723 screening-programme), together with data from the University of Cambridge symptomatic

724 testing programme (https://www.cam.ac.uk/coronavirus/stay-safe-cambridge-uni/data-from-

725 covid-19-testing-service). Reasonable requests for additional de-identified data will be

726 facilitated by the authors, provided they are in accordance with the terms of consent and

727 ethical approval, and relevant legal and regulatory requirements (such as, relating to data

728 protection and privacy).

729

730 Code availability

731 The code used for the model-based analysis included in this manuscript can be found at:

732 https://github.com/magicicada/covid19-caribbean-educational-

733 model/tree/manuscript cambs testing scheme.

734

735 Acknowledgements

736 NJM is supported by the MRC (CSF MR/P008801/1) and NHSBT (WPA15-02). RJS is supported by the EPSRC (EP/P031447/1 and EP/N031938/1). MPW was funded by the

738 Wellcome Trust through a Senior Clinical Research Fellowship (108070/Z/15/Z),

739 Addenbrooke's Charitable Trust and the NIHR Cambridge Biomedical Research Centre. BW

740 and AC receive funding from the National Institute for Health Research Cambridge 
741 Biomedical Research Centre at the Cambridge University Hospitals NHS Foundation Trust.

742 DA is a Wellcome Clinical PhD Fellow and gratefully supported by the Wellcome Trust

$743(222903 / Z / 21 / Z)$. VSB receives funding from the National Institute for Health Research at the

744 University of Cambridge. PJL was funded by the Wellcome Trust through a Principal

745 Research Fellowship (210688/Z/18/Z), the MRC (MR/V011561/1), the Addenbrooke's

746 Charitable Trust and the NIHR Cambridge Biomedical Research Centre. SB was funded by

747 the Wellcome Trust through a Senior Research Fellowship (215515/Z/19/Z). IG is a

748 Wellcome Senior Fellow and supported by funding from the Wellcome Trust (207498/Z/17/Z

749 and 206298/B/17/Z). We thank members of NHS Test and Trace and the Public Health

750 England contact tracing data team for their contributions to this study. We are grateful to all

751 staff members of the Cambridge COVID-19 Testing Centre for generating qPCR data. We

752 thank all students at the University of Cambridge who participated in screening, as well as

753 the College and University staff who have supported the programme.

754

755 Author contributions

$756 \mathrm{NM}$ designed the screening programme, with input from $\mathrm{PL}, \mathrm{MW}, \mathrm{BW}, \mathrm{PM}, \mathrm{DM}, \mathrm{RH}, \mathrm{KL}, \mathrm{VS}$

757 and $\mathrm{JH}$. NM is lead $\mathrm{PI}$ for the screening programme. BW is clinical lead for the screening

758 programme. PM chairs the oversight committee for the screening programme. DM is

759 logistics lead for the screening programme. MW is lead PI for the symptomatic testing

760 programme. SF is nursing lead for the screening programme. RH led the COVID-19 testing

761 facility. KL advised on legal and regulatory compliance. VS and JH developed IT systems.

762 MM led the telephone symptoms survey. All authors assisted with programme

763 implementation and data collection. BW cleaned the data. BW, NM and JE analysed the

764 data. JE performed modelling. RS advised on statistical analysis. NM and BW drafted the

765 manuscript, with input from JE and editing and approval from all authors. 


\section{Competing interests}

$768 \mathrm{RH}$ and SR are employees of AstraZeneca AB. RC is an employee of Charles River

769 Laboratories. All other authors declare no competing interests relevant to the submitted

770 work. 


\section{Figures}

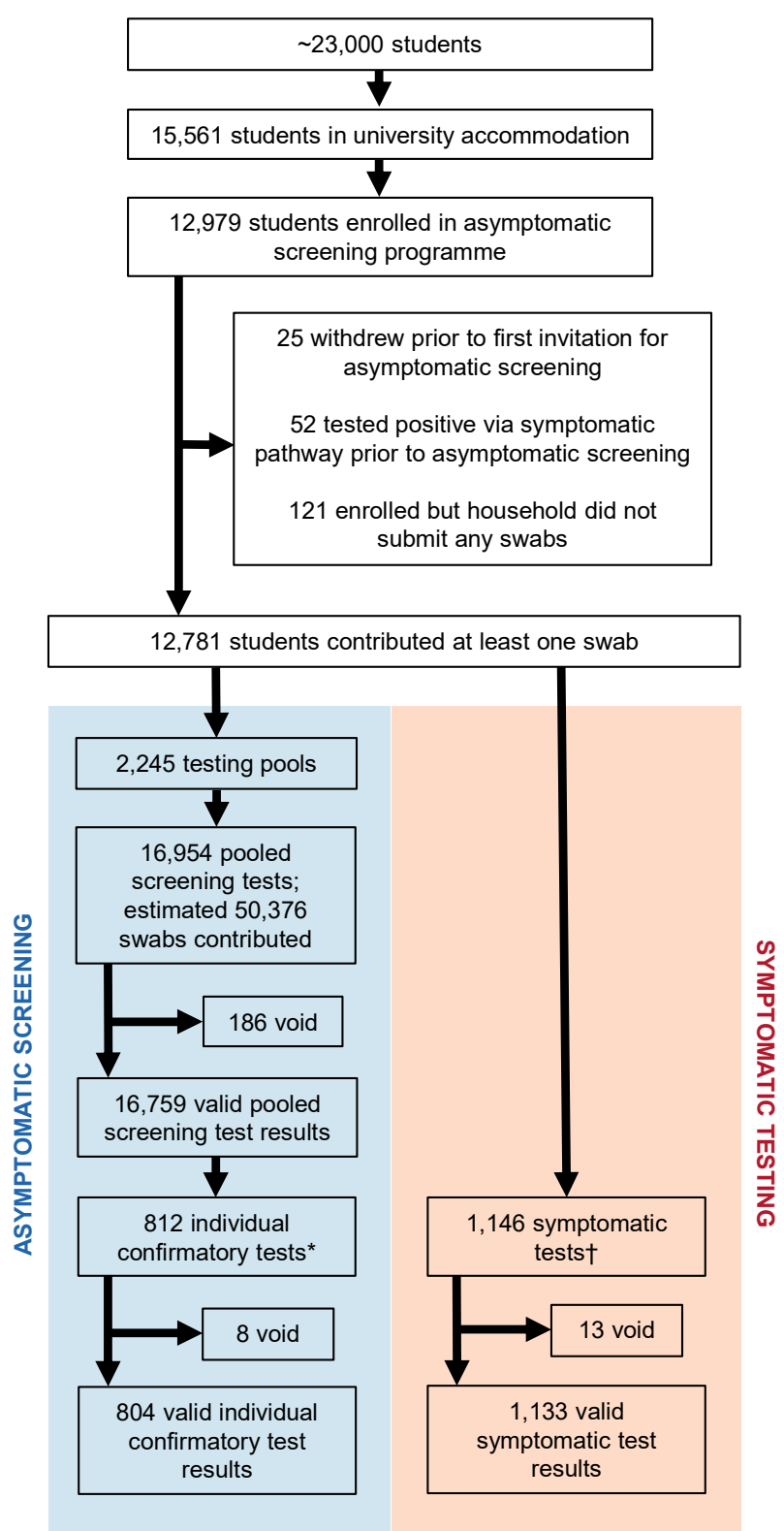

772

773 Fig. 1: Study cohort.

774 Participation in University of Cambridge SARS-CoV-2 asymptomatic screening programme

775 and symptomatic testing by students living in university accommodation from October 5 ,

7762020 to December 5, 2020. *Includes 19 repeat individual confirmatory tests. †Excludes

777 symptomatic tests conducted by NHS pathways. 


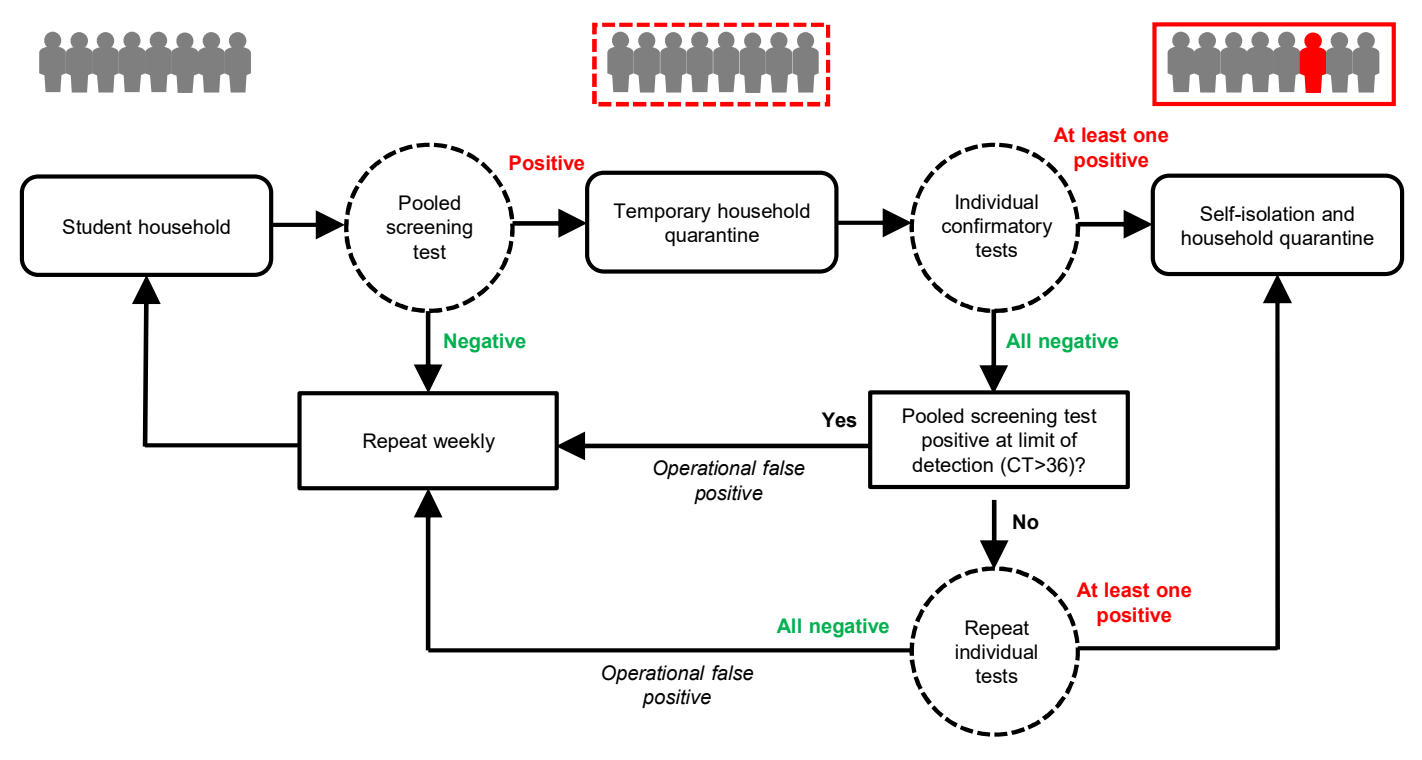

779 Fig. 2: Schematic overview of asymptomatic screening programme.

780 Pools of students, generally corresponding with student households, were screened weekly.

781 In the event of a positive pooled screening test, follow-up individual confirmatory tests were

782 used to identify the infected student or students. If all individual confirmatory tests were

783 negative, students were released from self-isolation. CT, cycle threshold. Operational false

784 positive, a positive pooled screening test followed by negative individual confirmatory tests

785 from all students in the testing pool. 
a

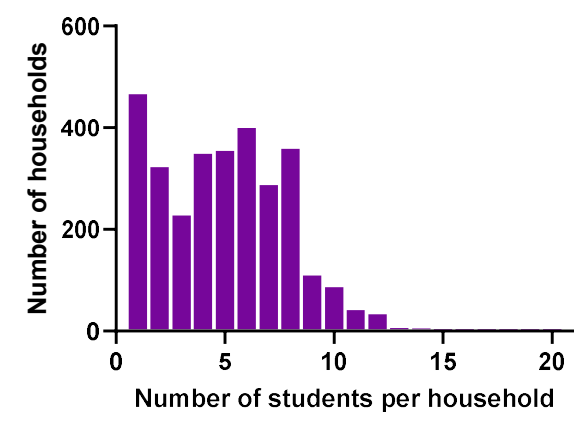

C

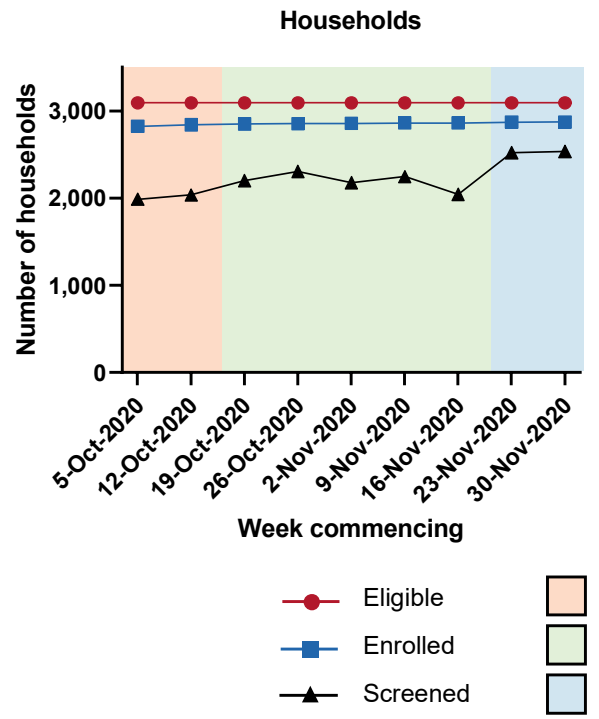

b

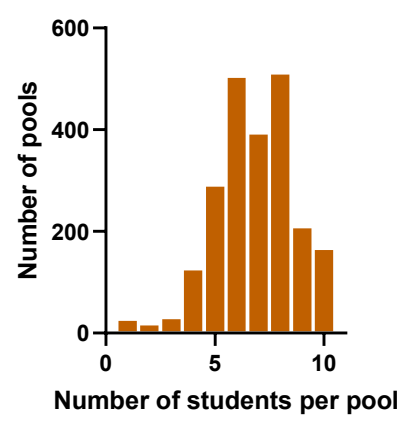

d

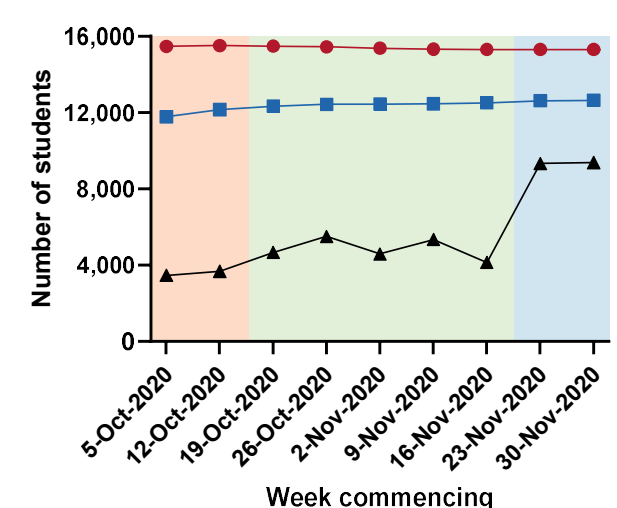

Two students per testing pool screened each week

Half of each testing pool screened on alternating weeks

Whole testing pool screened each week

e

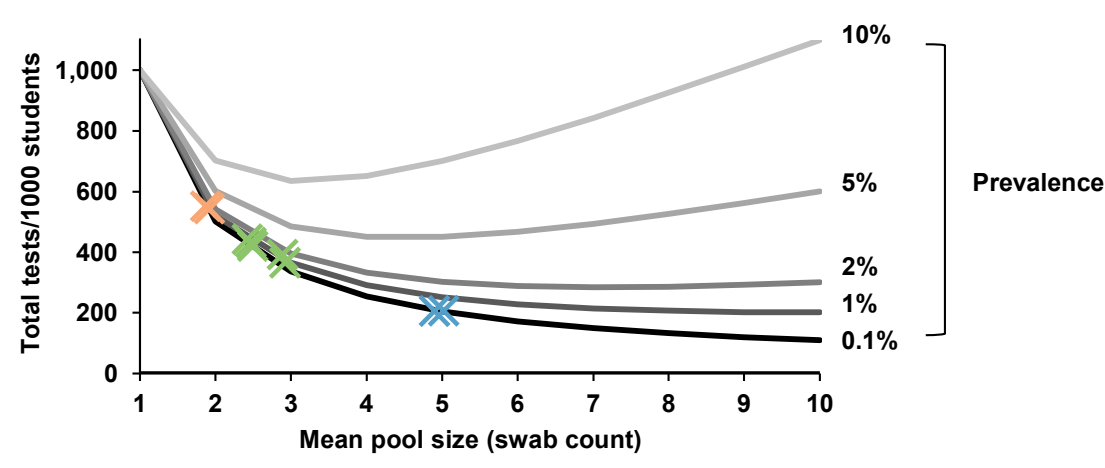

×Weeks 1-2 ×Weeks 3-7 $\times$ Weeks 8-9

787 Fig. 3: Student participation and implementation of swab pooling.

788 a-b, Distribution of (a) household size and (b) testing pool size for eligible students in

789 university accommodation. c-d, Weekly participation rates during autumn term by household

790 (c) and individual student (d). The number of eligible (red circles), enrolled (blue squares)

791 and screened (black triangles) students and households is shown during the phased implementation of the screening programme (indicated by the shaded areas in each plot). 
793 Some eligible students were excluded from screening each week because of a recent

794 positive test for SARS-CoV-2, symptoms of possible COVID-19, or a requirement to self-

795 isolate. e, Predicted total number of pooled screening and individual confirmatory tests

796 required to screen 1,000 students in the presence of different testing pool sizes and SARS-

797 CoV-2 prevalences (grey lines, 0.1-10\%). Crosses indicate the actual mean pool sizes and

798 tests utilised at the indicated time points. In the absence of swab pooling (equivalent to a

799 mean pool size of 1), 1,000 tests would be required. 
a

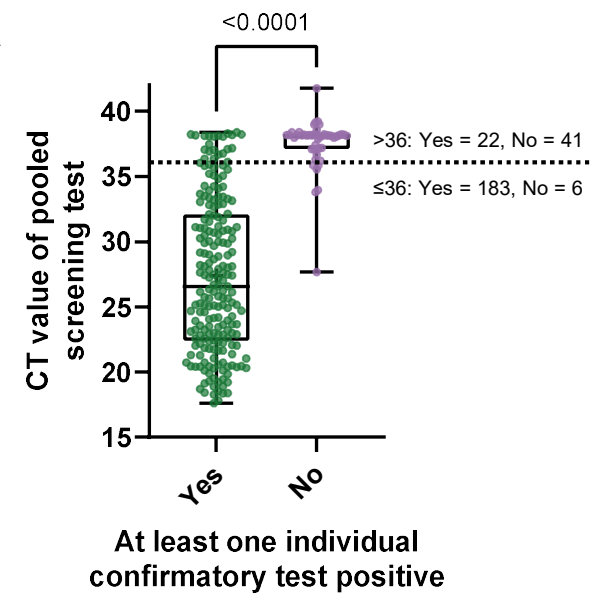

b

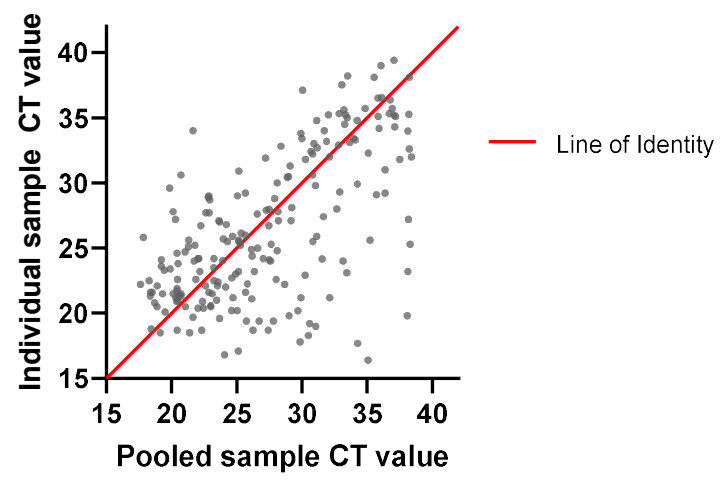

C

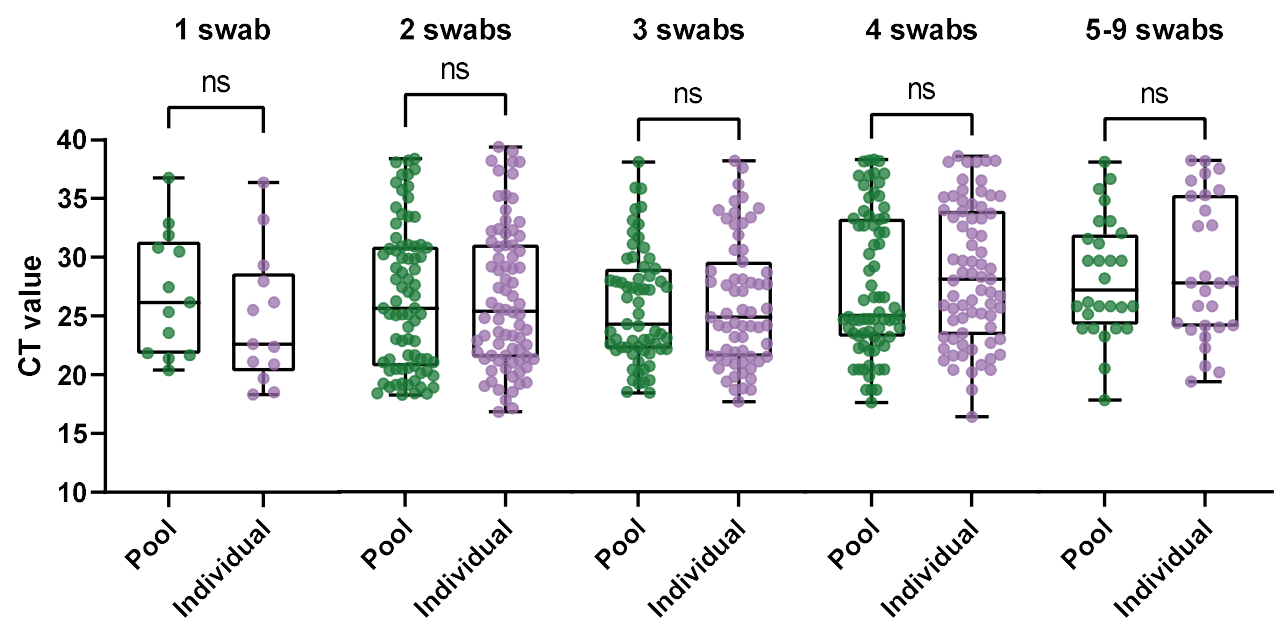

800

801 Fig. 4: Clinical validation of swab pooling.

802 a, SARS-CoV-2 cycle threshold (CT) values for pooled screening tests, comparing 203 pooled 803 samples resulting in positive individual confirmatory tests with 47 operational false positives 804 (no positive individual confirmatory tests). Centre line indicates median, boxes indicate 805 interquartile range, whiskers indicate range. Distributions were compared using an unpaired 806 2-tailed $t$-test. b, Comparison of CT values between positive paired pooled screening and 807 individual confirmatory tests. Where a positive pooled screening test resulted in $>1$ positive 808 individual confirmatory test, the lowest individual CT value is plotted. c, Comparison of CT 809 values between positive paired pooled screening and individual confirmatory tests, stratified 810 by number of swabs in the pooled sample. Distributions were compared using paired 2-tailed $811 t$-tests. ns, not significant, $P>0.05$. 
a

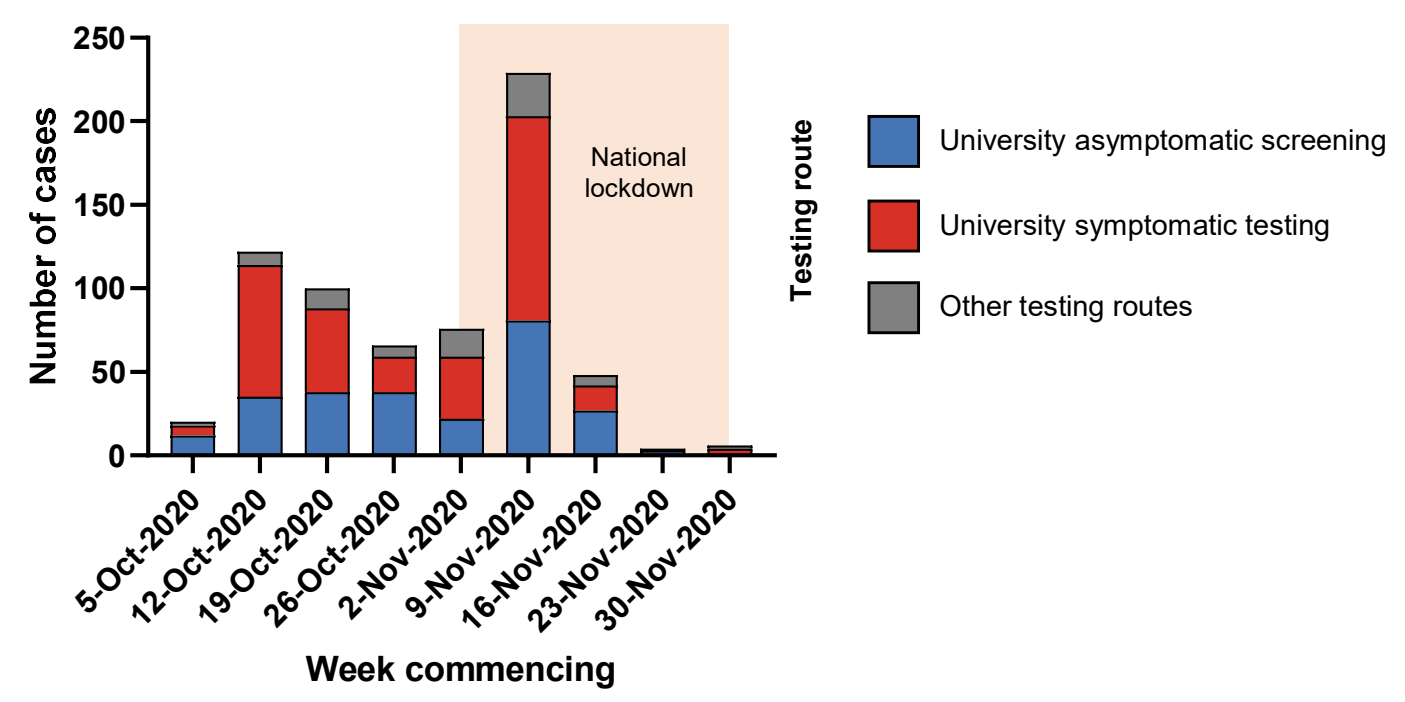

b

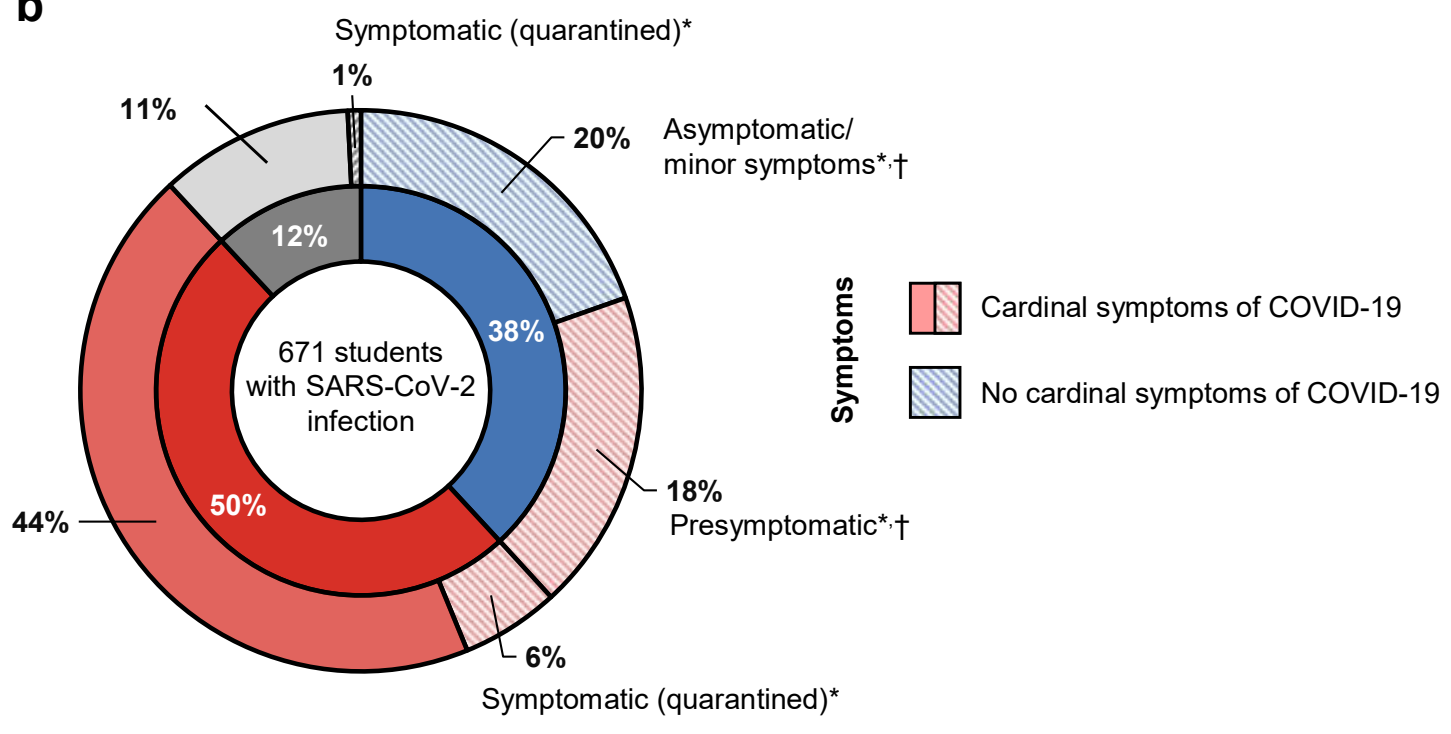

Fig. 5. Case ascertainment by asymptomatic screening and symptomatic testing.

814 a, Epidemic curve of weekly SARS-CoV-2 cases amongst participating students identified by 815 asymptomatic screening (blue), symptomatic testing (red) or other testing routes (grey). The 816 steep decline in cases coinciding with the second UK national lockdown is indicated by the 817 shaded area.

818 b, Classification of SARS-CoV-2 cases amongst participating students according to testing 819 pathway (inner doughnut) and symptoms (outer doughnut). Inner doughnut: same colours as 820 (a). Outer doughnut: red, cardinal symptoms of SARS-CoV-2 (fever, cough and/or 
821 anosmia/ageusia) experienced at some time during infection; blue, no symptoms or minor 822 symptoms only (no cardinal symptoms). *Cases where potential for onward transmission 823 was interrupted by asymptomatic screening are indicated by patterned areas. †Estimated 824 numbers based on retrospective telephone survey (sampling 140/256 students). 
a
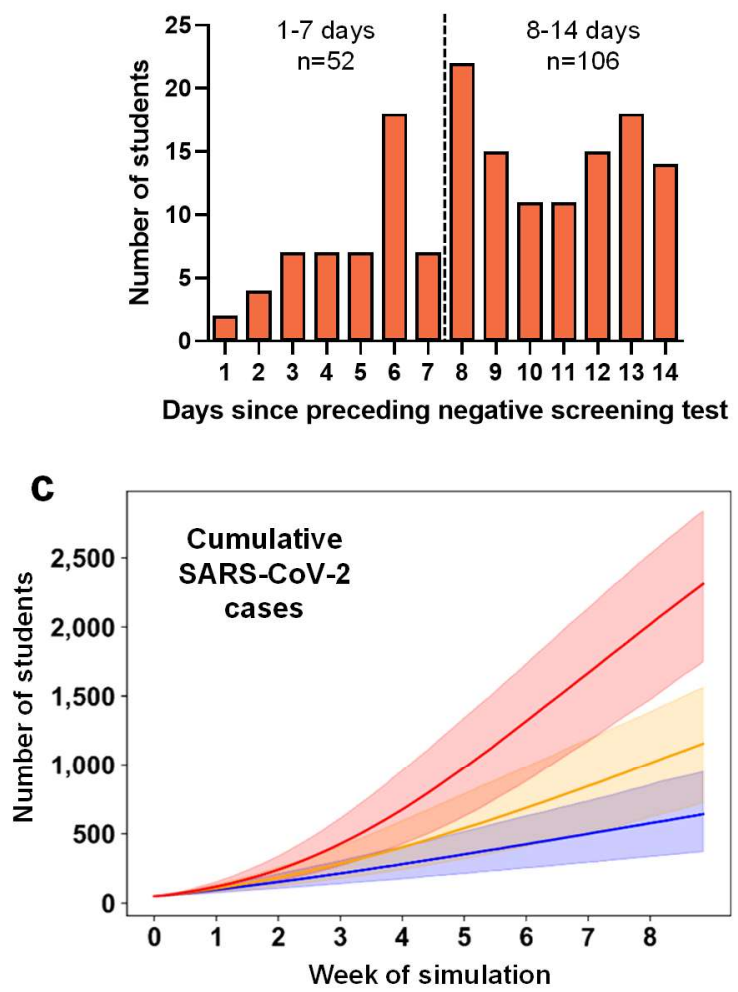

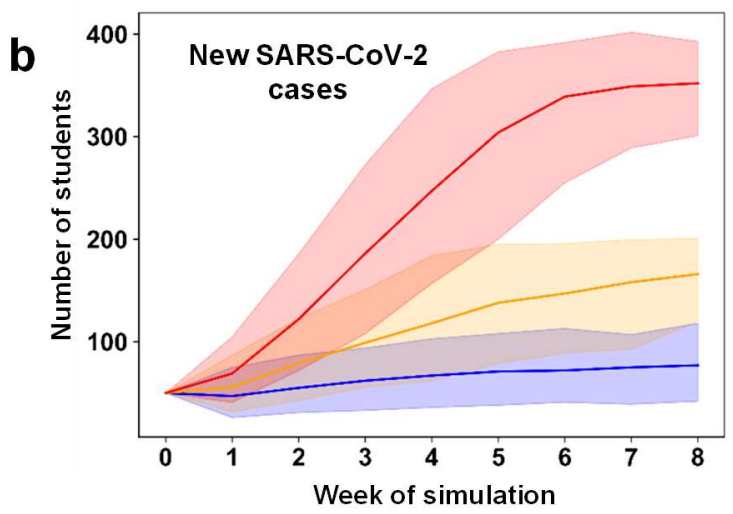

d

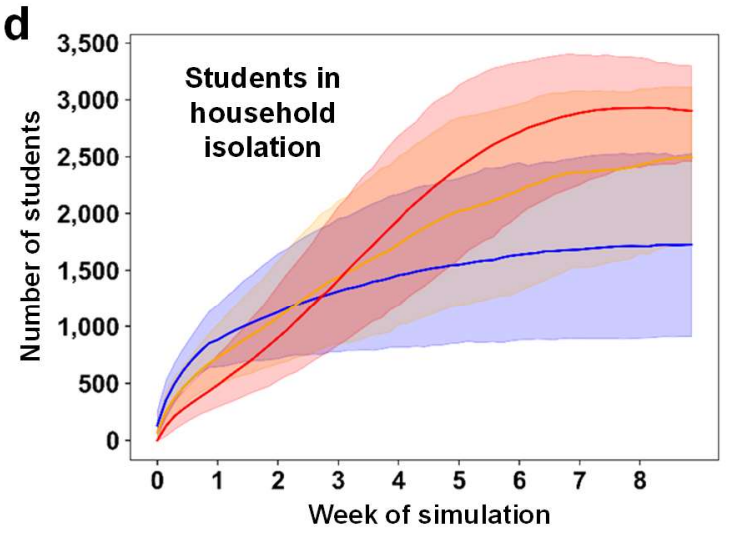

826 Fig. 6. Efficacy of asymptomatic screening.

827 a, Time between positive symptomatic test and most recent negative screening test for 158

828 students with symptomatic SARS-CoV-2 infection identified during weeks 3 to 7 . Half of each

829 testing pool was screened on alternating weeks over this period, corresponding to a

830 screening interval of 14 days for individual students. b-d, Model outputs indicating numbers

831 of new SARS-CoV-2 cases (a), cumulative SARS-CoV-2 cases (b) and students in

832 household isolation (c) under different testing regimens. Solid lines, median values; shaded

833 areas, 95\% confidence intervals. 


\section{Extended Data Figures}

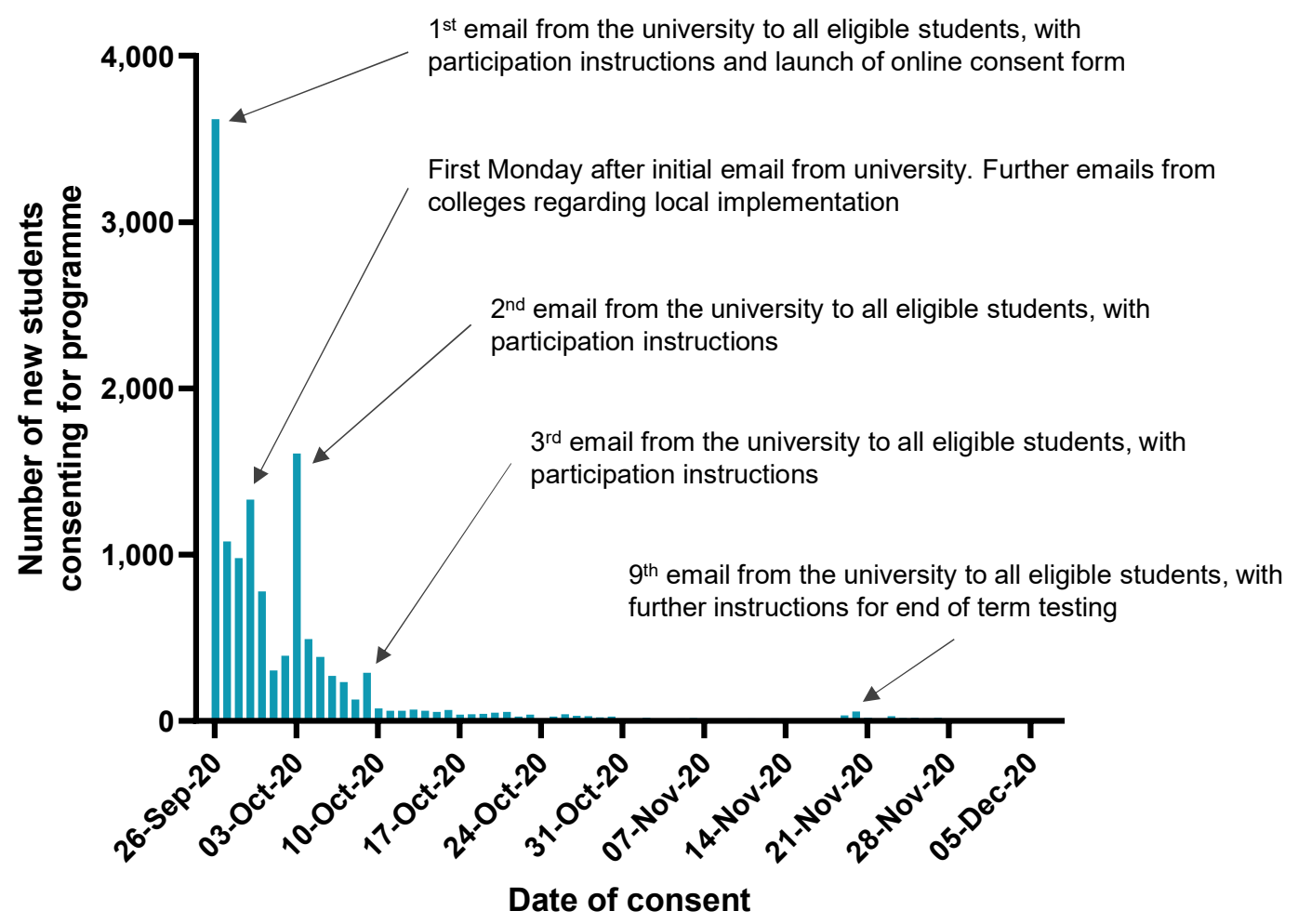

835

836 Extended Data Fig. 1: Impact of communications on study participation.

837 Daily number of students consenting to participate in asymptomatic screening programme.

838 Key communications are highlighted. Where students consented more than once, only their first consent is included. 
1 swab

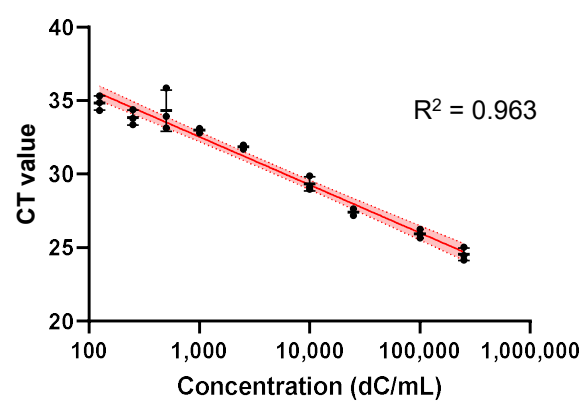

5-8 swabs

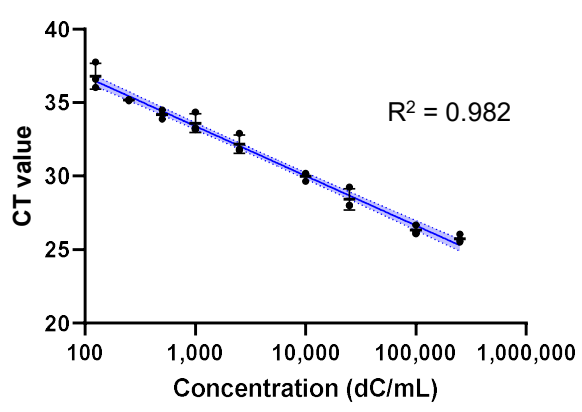

840

\section{Extended Data Fig. 2: Technical validation of swab pooling.}

842 Detection of SARS CoV-2 standards in reconstituted individual (1 swab) or pooled (5-8

843 swabs) samples. Experiment conducted in triplicate, with individual data points, mean and

844 standard deviation shown for each SARS-CoV-2 concentration. $\mathrm{dC} / \mathrm{mL}$, digital copies $/ \mathrm{mL}$.

845 CT value, cycle threshold. Solid lines, non-linear regression lines; shaded areas, 95\%

846 confidence intervals. 
a

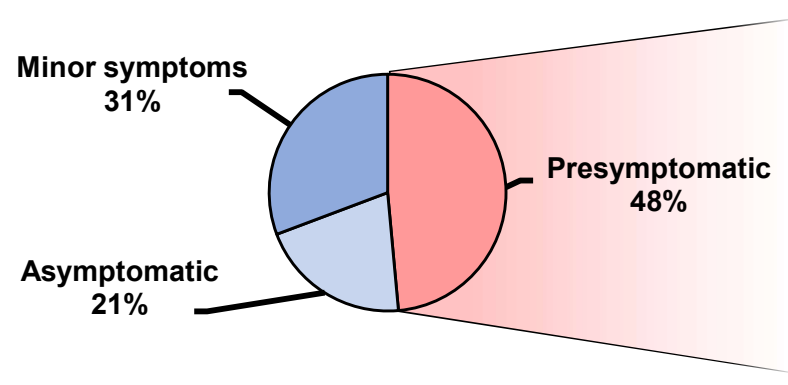

C

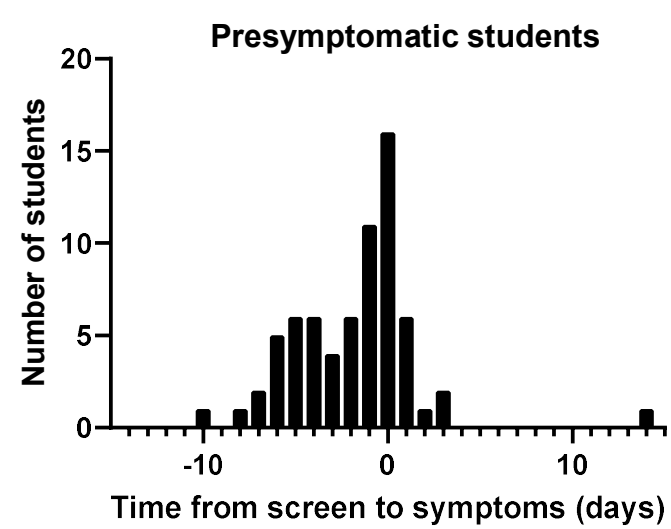

b

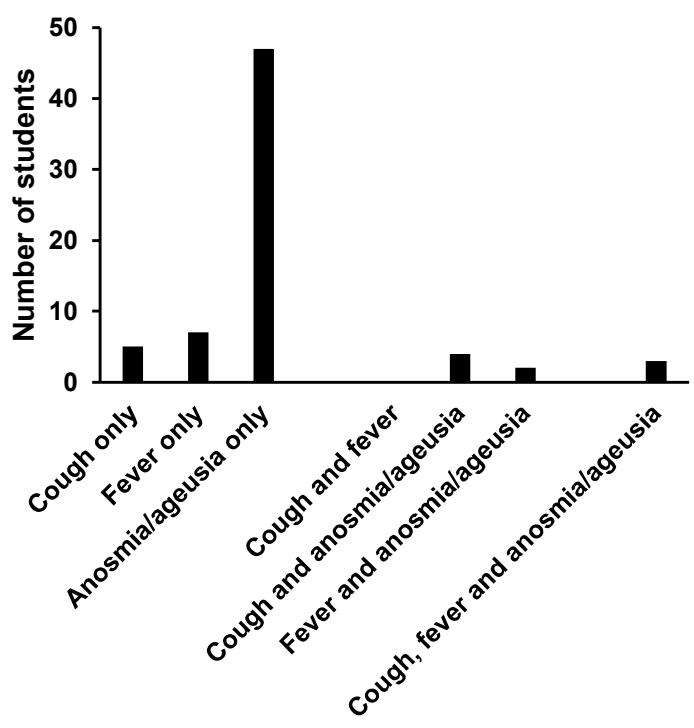

847

848 Extended Data Fig. 3: Symptoms identified by telephone survey.

849 a, Proportion of students identified by asymptomatic screening who went on to develop 850 cardinal symptoms of COVID-19 (fever, cough, and/or anosmia/ageusia) at some time during infection (presymptomatic, red), those who experienced no symptoms at all (asymptomatic, pale blue), and those who reported only minor symptoms (minor symptoms, dark blue). Data are shown for 140 students who responded to the retrospective telephone survey. b. Frequency of individual symptoms reported by 68 presymptomatic students from (a). c, Time interval between positive screening test and onset of cardinal symptoms of COVID-19 for 68 presymptomatic students from (a). Since presymptomatic students may be sampled at any time during their presymptomatic infectious period, and (on average) halfway through, the mean presymptomatic infectious period is expected to be twice the mean observed time interval. 
a

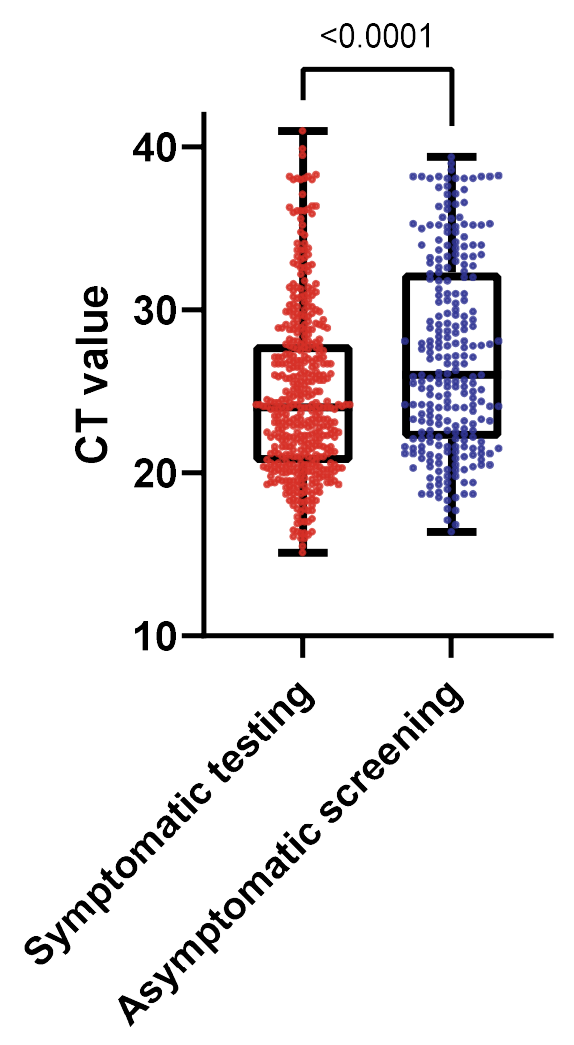

b
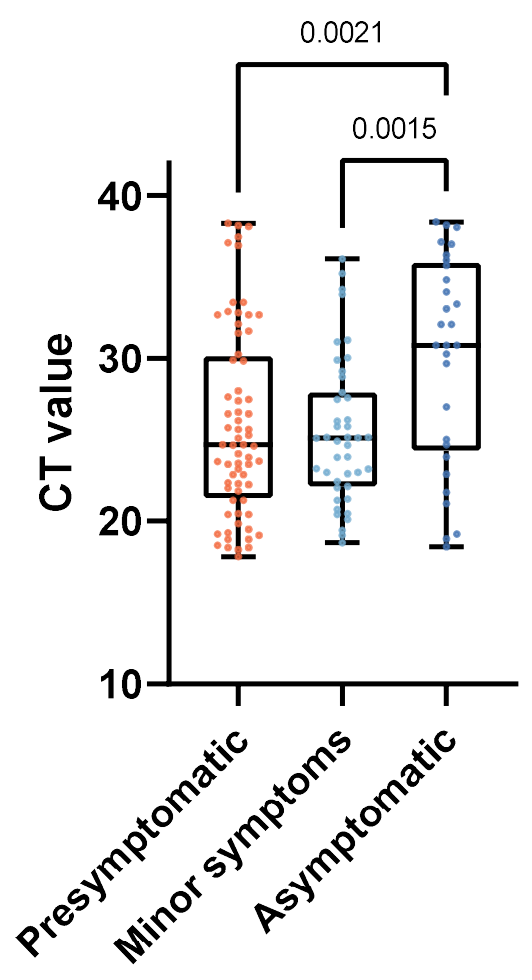

860

861 Extended Data Fig. 4: Comparison of CT values by testing pathway and symptoms.

862 a, SARS-CoV-2 cycle threshold (CT) values for positive university symptomatic tests, compared with positive individual confirmatory tests from the asymptomatic screening programme. $\mathbf{b}$, Comparison of CT values for students identified by asymptomatic screening who went on to develop cardinal symptoms of COVID-19 (fever, cough, and/or anosmia/ageusia) at some time during infection (presymptomatic, red), those who experienced no symptoms at all (asymptomatic, dark blue), and those who reported only

868 minor symptoms (minor symptoms, pale blue). Data are shown for 140 students who responded to the retrospective telephone survey. Centre line indicates median, boxes

870 indicate interquartile range, whiskers indicate range. $P$ values were calculated for pairwise 871 comparisons using unpaired two-tailed $t$-tests (a) and ordinary one-way ANOVA (b). 
a

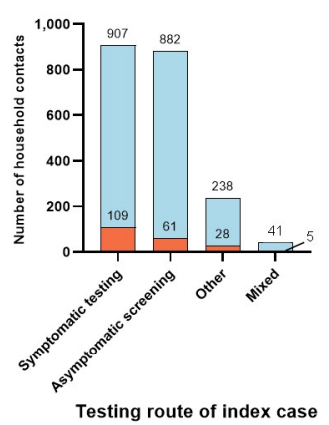

b

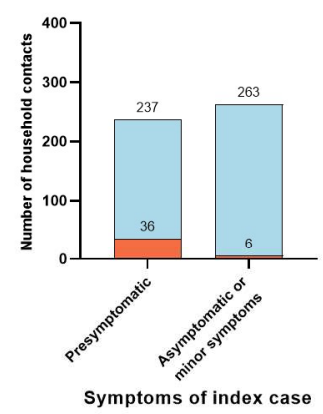

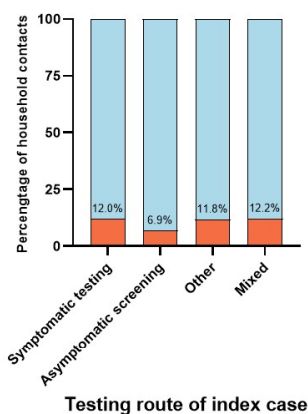

$\square$ No positive test within 14 days of index

$\square$ Positive test within 14 days of index

C

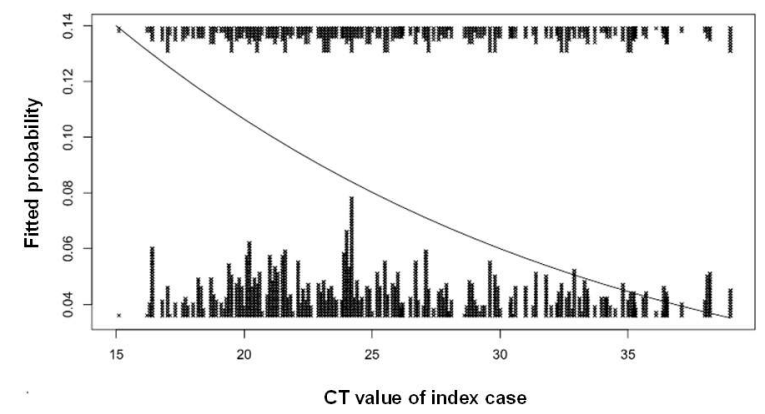

872

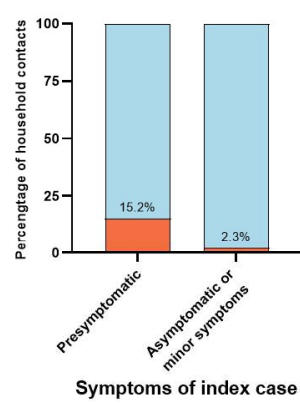

$\square$ No positive test within 14 days of index

$\square$ Positive test within 14 days of index

d
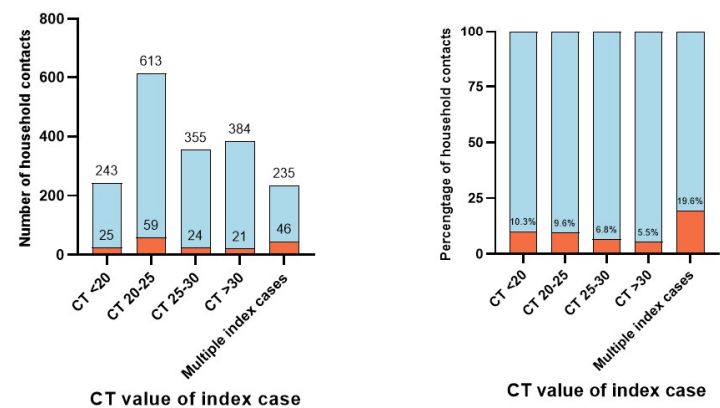

$\square$ No positive test within 14 days of index

$\square$ Positive test within 14 days of index

873 Extended Data Fig. 5: Secondary household attack rates.

874 a, Total number (left panel) and proportion (right panel) of household contacts of students

875 with confirmed SARS-CoV-2 infection who tested positive for SARS-CoV-2 within 2 weeks of

876 the index case (any testing pathway). Total household contacts (orange plus blue bars) and

877 household contacts testing positive for SARS-CoV-2 (orange bars) are shown. Data are 
878 stratified by testing pathway of the index cases. Other, students identified from notifications

879 to Public Health England. Mixed, multiple students from same household identified on the 880 same index day, using different testing pathways. The proportion of household contacts who 881 tested positive was significantly different for asymptomatic (6.9\%) versus symptomatic $882(12.0 \%)$ index cases $(P=0.0003$, Fisher's exact test). $\mathbf{b}$, As for $(\mathbf{a})$, but results are shown for 883140 index cases identified by asymptomatic screening who responded to the retrospective 884 telephone survey. Data are stratified according to whether index cases went on to develop 885 cardinal symptoms of COVID-19 (fever, cough, and/or anosmia/ageusia) at some time 886 during infection (presymptomatic), or remained asymptomatic/reported only minor symptoms 887 (asymptomatic or minor symptoms). The proportion of household contacts who tested 888 positive was significantly different for contacts of presymptomatic index cases $(15.2 \%)$ 889 versus contacts of index cases who remained asymptomatic/reported only minor symptoms $890(2.3 \%)(P<0.0001$, Fisher's exact test). c, Fitted probability of a household contact testing 891 positive within 14 days of an index case testing positive, as a function of the cycle threshold 892 (CT) value of the index case (solid line). The estimated regression coefficient corresponding 893 to the index case CT value was -0.06239 . Testing whether this coefficient was zero yielded 894 a $P<0.001$. Positive and negative household contacts are plotted as crosses at the top and 895 bottom of the plot, respectively. Data are shown for 1,595 household contacts, excluding 896 households where multiple students were identified on the same index day. $\mathbf{d}$, As for (a), but 897 data are stratified according to CT value of the index case. Where multiple students from the 898 same household were identified on the same index day, data are shown separately (multiple 899 index cases). 
a: Number of non-household activity groups
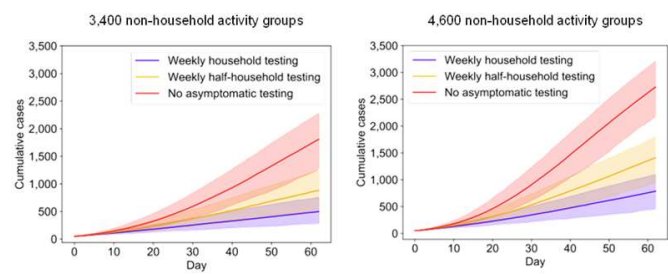

b: Proportion symptomatic
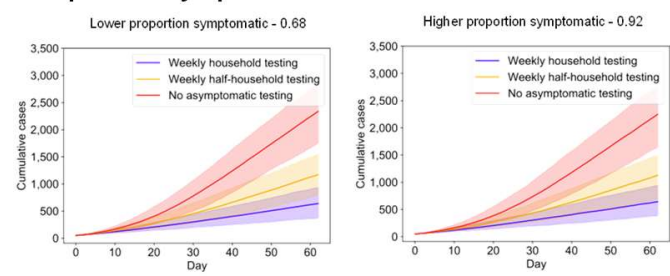

c: Probability of transmission for a household contact per day
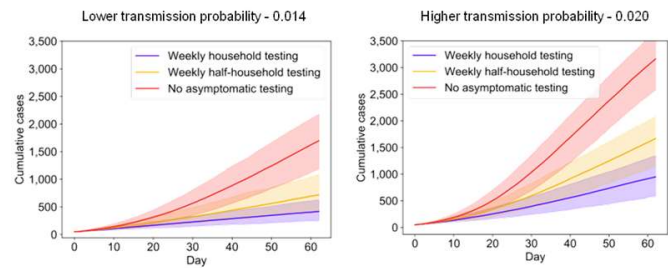

d: Ratio of probability of transmission for a nonhousehold contact per day to probability of transmission for a household contact per day
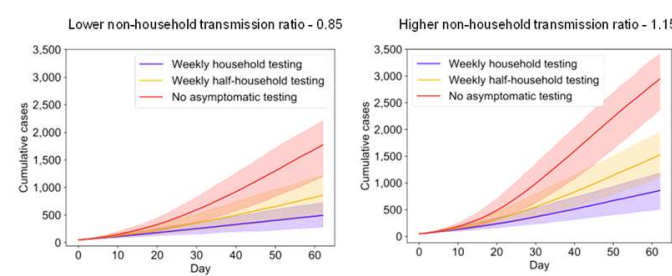

900

\section{Extended Data Fig. 6: Sensitivity analyses.}

902 Sensitivity analyses for model outputs indicating numbers of cumulative SARS-CoV-2 cases

903 under different testing regimens. In each case, the indicated parameter is decreased or

904 increased by $15 \%$. No asymptomatic testing, no asymptomatic screening; weekly half-

905 household testing, half of each testing pool screened on alternating weeks; weekly

906 household testing, whole testing pool screened each week.
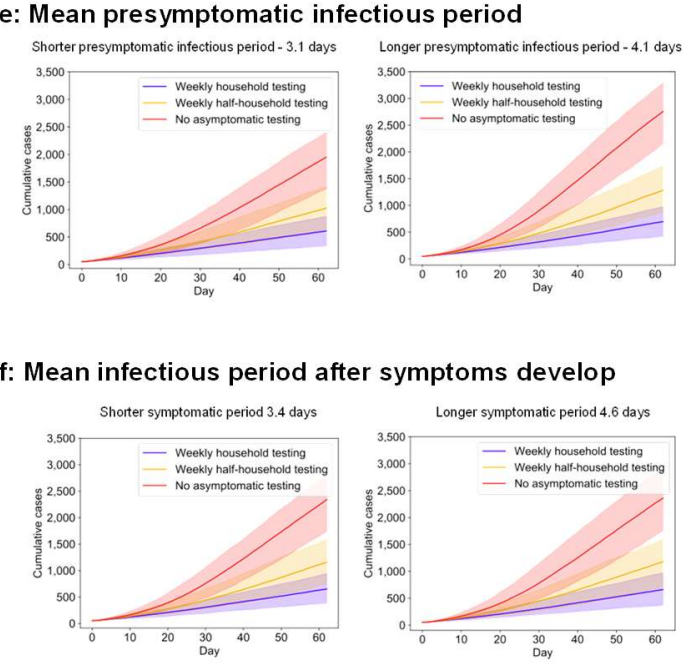

g: Ratio of infectiousness of asymptomatics (compared to symptomatics)

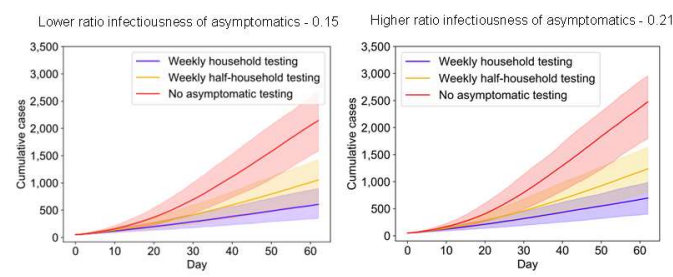

f: Mean infectious period after symptoms develop 


\section{References}

9081 Ferretti, L. et al. Quantifying SARS-CoV-2 transmission suggests epidemic control

909 with digital contact tracing. Science 368, doi:10.1126/science.abb6936 (2020).

9102 Hellewell, J. et al. Feasibility of controlling COVID-19 outbreaks by isolation of cases

911 and contacts. Lancet Glob Health 8, e488-e496, doi:10.1016/S2214-109X(20)30074-

912 7 (2020).

913

Kretzschmar, M. E. et al. Impact of delays on effectiveness of contact tracing strategies for COVID-19: a modelling study. The Lancet Public Health 5, e452-e459 (2020).

915

916

Oran, D. P. \& Topol, E. J. The proportion of SARS-CoV-2 infections that are

917 asymptomatic: a systematic review. Ann Intern Med, doi: 10.7326/M7320-6976

918 (2021).

Tabata, S. et al. Clinical characteristics of COVID-19 in 104 people with SARS-CoV-2 infection on the Diamond Princess cruise ship: a retrospective analysis. Lancet Infect

921

922 Dis 20, 1043-1050, doi:10.1016/S1473-3099(20)30482-5 (2020).

923

924

925

926

927

Wang, Y. et al. Clinical Outcomes in 55 Patients With Severe Acute Respiratory Syndrome Coronavirus 2 Who Were Asymptomatic at Hospital Admission in Shenzhen, China. J Infect Dis 221, 1770-1774, doi:10.1093/infdis/jiaa119 (2020).

7 Johansson, M. A. et al. SARS-CoV-2 Transmission From People Without COVID-19 Symptoms. JAMA Network Open 4, e2035057-e2035057, doi:10.1001/jamanetworkopen.2020.35057 (2021).

8 Buitrago-Garcia, D. et al. Occurrence and transmission potential of asymptomatic and presymptomatic SARS-CoV-2 infections: A living systematic review and metaanalysis. PLoS Med 17, e1003346, doi:10.1371/journal.pmed.1003346 (2020).

9 Paltiel, A. D., Zheng, A. \& Walensky, R. P. Assessment of SARS-CoV-2 Screening Strategies to Permit the Safe Reopening of College Campuses in the United States. JAMA Netw Open 3, e2016818, doi:10.1001/jamanetworkopen.2020.16818 (2020).

934

Peto, J. et al. Weekly COVID-19 testing with household quarantine and contact tracing is feasible and would probably end the epidemic. Royal Society Open Science 7, 200915 (2020).

937

11 Grassly, N. C. et al. Comparison of molecular testing strategies for COVID-19

939 control: a mathematical modelling study. Lancet Infect Dis 20, 1381-1389, doi:10.1016/S1473-3099(20)30630-7 (2020).

Rivett, L. et al. Screening of healthcare workers for SARS-CoV-2 highlights the role of asymptomatic carriage in COVID-19 transmission. Elife 9, doi:10.7554/eLife.58728 (2020).

World Health Organization. Infection prevention and control guidance for long-term care facilities in the context of COVID-19: interim guidance, 21 March 2020, 
94614 Centers for Disease Control and Prevention. Interim infection prevention and control recommendations for healthcare personnel during the coronavirus disease 2019 (COVID-19) pandemic, <https://www.cdc.gov/coronavirus/2019-ncov/hcp/infectioncontrol.html> (2020).

15 Jones, N. K. et al. Effective control of SARS-CoV-2 transmission between healthcare workers during a period of diminished community prevalence of COVID-19. Elife $\mathbf{9}$, doi:10.7554/eLife.59391 (2020).

Hagan, L. M. et al. Mass Testing for SARS-CoV-2 in 16 Prisons and Jails - Six Jurisdictions, United States, April-May 2020. MMWR Morb Mortal Wkly Rep 69, 1139-1143, doi:10.15585/mmwr.mm6933a3 (2020).

Kissler, S. M. et al. Viral dynamics of SARS-CoV-2 infection and the predictive value of repeat testing. , <Preprint at

https://www.medrxiv.org/content/medrxiv/early/2020/10/23/2020.10.21.20217042.full. pdf> (2020).

959

960

18 Torjesen, I. What do we know about lateral flow tests and mass testing in schools?

961

962 BMJ 372:n706 (2021).

963

lacobucci, G. Covid-19: Government rolls out twice weekly rapid testing to all in England. BMJ doi: 10.1136/bmj.n902 (2021).

965

Raffle, A. E., Pollock, A. M. \& Harding-Edgar, L. Covid-19 mass testing programmes. BMJ 370, m3262, doi:10.1136/bmj.m3262 (2020).

967

968

969

970

Gill, M. \& Gray, M. Mass testing for covid-19 in the UK. BMJ 371, m4436, doi:10.1136/bmj.m4436 (2020).

22 Wilson, E. et al. Multiple COVID-19 Clusters on a University Campus - North Carolina, August 2020. MMWR Morb Mortal Wkly Rep 69, 1416-1418, doi:10.15585/mmwr.mm6939e3 (2020).

976

977

Fox, M. D., Bailey, D. C., Seamon, M. D. \& Miranda, M. L. Response to a COVID-19 Outbreak on a University Campus - Indiana, August 2020. MMWR Morb Mortal Wkly Rep 70, 118-122, doi:10.15585/mmwr.mm7004a3 (2021).

Vang, K. E. et al. Participation in Fraternity and Sorority Activities and the Spread of COVID-19 Among Residential University Communities - Arkansas, August 21-

September 5, 2020. MMWR Morb Mortal Wkly Rep 70, 20-23, doi:10.15585/mmwr.mm7001a5 (2021).

Davies, N. G. et al. Age-dependent effects in the transmission and control of COVID19 epidemics. Nature Medicine 26, 1205-1211, doi:10.1038/s41591-020-0962-9 (2020).

26 Poletti, P. et al. Association of Age With Likelihood of Developing Symptoms and Critical Disease Among Close Contacts Exposed to Patients With Confirmed SARSCoV-2 Infection in Italy. JAMA Netw Open 4, e211085, doi:10.1001/jamanetworkopen.2021.1085 (2021).

Benneyan, J., Gehrke, C., Ilies, I. \& Nehls, N. Community and Campus COVID-19 Risk Uncertainty Under University Reopening Scenarios: Model-Based Analysis. JMIR Public Health Surveill 7, e24292, doi:10.2196/24292 (2021). 
28 Yamey, G. \& Walensky, R. P. Covid-19: re-opening universities is high risk. BMJ 370, m3365, doi:10.1136/bmj.m3365 (2020).

29 Gillam, T. B. et al. Norwich COVID-19 testing initiative pilot: evaluating the feasibility of asymptomatic testing on a university campus. J Public Health (Oxf) 43, 82-88, doi:10.1093/pubmed/fdaa194 (2021).

30 Blake, H. et al. Perceptions and Experiences of the University of Nottingham Pilot SARS-CoV-2 Asymptomatic Testing Service: A Mixed-Methods Study. Int J Environ Res Public Health 18, doi:10.3390/ijerph18010188 (2020).

31 Denny, T. N. et al. Implementation of a Pooled Surveillance Testing Program for Asymptomatic SARS-CoV-2 Infections on a College Campus - Duke University, Durham, North Carolina, August 2-October 11, 2020. MMWR Morb Mortal Wkly Rep 69, 1743-1747, doi:10.15585/mmwr.mm6946e1 (2020).

32 Hamer, D. H. et al. Control of COVID-19 transmission on an urban university campus during a second wave of the pandemic. medRxiv, 2021.2002.2023.21252319, doi:10.1101/2021.02.23.21252319 (2021).

33 Rennert, L. et al. Surveillance-based informative testing for detection and containment of SARS-CoV-2 outbreaks on a public university campus: an observational and modelling study. Lancet Child Adolesc Health, doi:10.1016/S23524642(21)00060-2 (2021).

34 Hemani, G. et al. Modelling pooling strategies for SARS-CoV-2 testing in a university setting [version 1; peer review: awaiting peer review]. Wellcome Open Research 6, doi:10.12688/wellcomeopenres.16639.1 (2021).

35 Hill, E. M., Atkins, B. D., Keeling, M. J., Tildesley, M. J. \& Dyson, L. Modelling SARSCoV-2 transmission in a UK university setting. medRxiv, 2020.2010.2015.20208454, doi:10.1101/2020.10.15.20208454 (2021).

36 Pray, I. W. et al. Performance of an Antigen-Based Test for Asymptomatic and Symptomatic SARS-CoV-2 Testing at Two University Campuses - Wisconsin, September-October 2020. MMWR Morb Mortal Wkly Rep 69, 1642-1647, doi:10.15585/mmwr.mm695152a3 (2021).

37 Ford, L. et al. Epidemiologic characteristics associated with SARS-CoV-2 antigenbased test results, rRT-PCR cycle threshold values, subgenomic RNA, and viral culture results from university testing. Clin Infect Dis, doi:10.1093/cid/ciab303 (2021).

38 Barak, N. et al. Lessons from applied large-scale pooling of 133,816 SARS-CoV-2 RT-PCR tests. Sci Transl Med 13, doi:10.1126/scitranslmed.abf2823 (2021).

39 Ben-Ami, R. et al. Large-scale implementation of pooled RNA extraction and RTPCR for SARS-CoV-2 detection. Clinical Microbiology and Infection 26, 1248-1253 (2020).

40 Gupta, E. et al. Pooled RNA sample reverse transcriptase real time PCR assay for SARS CoV-2 infection: A reliable, faster and economical method. PLoS One 15, e0236859, doi:10.1371/journal.pone.0236859 (2020). 
41 Hogan, C. A., Sahoo, M. K. \& Pinsky, B. A. Sample Pooling as a Strategy to Detect Community Transmission of SARS-CoV-2. JAMA 323, 1967-1969, doi:10.1001/jama.2020.5445 (2020).

42 Lohse, S. et al. Pooling of samples for testing for SARS-CoV-2 in asymptomatic people. Lancet Infect Dis 20, 1231-1232, doi:10.1016/S1473-3099(20)30362-5 (2020).

43 Perchetti, G. A. et al. Pooling of SARS-CoV-2 samples to increase molecular testing throughput. J Clin Virol 131, 104570, doi:10.1016/j.jcv.2020.104570 (2020).

44 Torres, I., Albert, E. \& Navarro, D. Pooling of nasopharyngeal swab specimens for SARS-CoV-2 detection by RT-PCR. J Med Virol 92, 2306-2307, doi:10.1002/jmv.25971 (2020).

45 Yelin, I. et al. Evaluation of COVID-19 RT-qPCR Test in Multi sample Pools. Clin Infect Dis 71, 2073-2078, doi:10.1093/cid/ciaa531 (2020).

46 Christoff, A. P. et al. Swab pooling: A new method for large-scale RT-qPCR screening of SARS-CoV-2 avoiding sample dilution. PLoS One 16, e0246544, doi:10.1371/journal.pone.0246544 (2021).

47 Thanh, T. T. et al. The Application of Sample Pooling for Mass Screening of SARSCoV-2 in an Outbreak of COVID-19 in Vietnam. Am J Trop Med Hyg, doi:10.4269/ajtmh.20-1583 (2021).

48 Chen, F. et al. Comparing two sample pooling strategies for SARS-CoV-2 RNA detection for efficient screening of COVID-19. J Med Virol 93, 2805-2809, doi:10.1002/jmv.26632 (2021).

49 Steel, K. \& Fordham, E. Coronavirus (COVID-19) Infection Survey pilot: England, Wales and Northern Ireland, 9 October 2020, Office for National Statistics, $<$ https://www.ons.gov.uk/peoplepopulationandcommunity/healthandsocialcare/conditi onsanddiseases/bulletins/coronaviruscovid19infectionsurveypilot/englandwalesandno rthernireland9october2020> (2020).

50 Medicines \& Healthcare products Regulatory Authority. Target Product Profile: Laboratory-Based SARS-CoV-2 Viral Detection tests, $<$ https://www.gov.uk/government/publications/how-tests-and-testing-kits-forcoronavirus-covid-19-work/target-product-profile-laboratory-based-sars-cov-2-viraldetection-tests> (2021).

51 Aggarwal, D. et al. Genomic epidemiology of SARS-CoV-2 in the University of Cambridge identifies dynamics of transmission. (2021).

52 Byrne, A. W. et al. Inferred duration of infectious period of SARS-CoV-2: rapid scoping review and analysis of available evidence for asymptomatic and symptomatic COVID-19 cases. BMJ Open 10, e039856, doi:10.1136/bmjopen-2020039856 (2020).

53 Wolfel, R. et al. Virological assessment of hospitalized patients with COVID-2019. Nature 581, 465-469, doi:10.1038/s41586-020-2196-x (2020). 
54 Hellewell, J. et al. Estimating the effectiveness of routine asymptomatic PCR testing at different frequencies for the detection of SARS-CoV-2 infections. BMC Med 19, 106, doi:10.1186/s12916-021-01982-x (2021).

55 Singanayagam, A. et al. Duration of infectiousness and correlation with RT-PCR cycle threshold values in cases of COVID-19, England, January to May 2020. Euro Surveill 25, doi:10.2807/1560-7917.ES.2020.25.32.2001483 (2020).

$56 \mathrm{He}, \mathrm{X}$. et al. Temporal dynamics in viral shedding and transmissibility of COVID-19. Nat Med 26, 672-675, doi:10.1038/s41591-020-0869-5 (2020).

57 Long, Q. X. et al. Clinical and immunological assessment of asymptomatic SARSCoV-2 infections. Nat Med 26, 1200-1204, doi:10.1038/s41591-020-0965-6 (2020).

58 Wolf, A., Hulmes, J. \& Hopkins, S. Lateral flow device specificity in phase 4 (post marketing) surveillance, <https://www.gov.uk/government/publications/lateral-flowdevice-specificity-in-phase-4-post-marketing-surveillance> (2021).

59 Woloshin, S., Patel, N. \& Kesselheim, A. S. False Negative Tests for SARS-CoV-2 Infection - Challenges and Implications. N Engl J Med 383, e38, doi:10.1056/NEJMp2015897 (2020).

60 Sehanobish, E. et al. COVID-19-Induced Anosmia and Ageusia Are Associated With Younger Age and Lower Blood Eosinophil Counts. Am J Rhinol Allergy, 19458924211004800, doi:10.1177/19458924211004800 (2021).

61 von Bartheld, C. S., Hagen, M. M. \& Butowt, R. Prevalence of Chemosensory Dysfunction in COVID-19 Patients: A Systematic Review and Meta-analysis Reveals Significant Ethnic Differences. ACS Chem Neurosci 11, 2944-2961, doi:10.1021/acschemneuro.0c00460 (2020).

$62 \mathrm{Li}, \mathrm{F}$. et al. Household transmission of SARS-CoV-2 and risk factors for susceptibility and infectivity in Wuhan: a retrospective observational study. Lancet Infect Dis 21, 617-628, doi:10.1016/S1473-3099(20)30981-6 (2021).

63 Berke, E. M. et al. Pooling-in-a-pod: A Strategy for COVID-19 Testing to Facilitate Safe Return to School. medRxiv, 2021.2003.2024.21254230, doi:10.1101/2021.03.24.21254230 (2021).

64 Cox $\mathrm{C}$ et al. Developing an ethical framework for asymptomatic COVID-19 testing programmes in higher education institutions [version 1; peer review: awaiting peer review]. Wellcome Open Res 6 (2021).

65 Mina, M. J., Peto, T. E., Garcia-Finana, M., Semple, M. G. \& Buchan, I. E. Clarifying the evidence on SARS-CoV-2 antigen rapid tests in public health responses to COVID-19. Lancet 397, 1425-1427, doi:10.1016/S0140-6736(21)00425-6 (2021).

66 Levine-Tiefenbrun, M. et al. Initial report of decreased SARS-CoV-2 viral load after inoculation with the BNT162b2 vaccine. Nat Med, doi:10.1038/s41591-021-01316-7 (2021).

67 Pavelka, M. et al. The impact of population-wide rapid antigen testing on SARS-CoV2 prevalence in Slovakia. Science, doi:10.1126/science.abf9648 (2021). 
Scientific Advisory Group for Emergencies. Innova Lateral Flow SARS-CoV-2 Antigen test accuracy in Liverpool Pilot: Preliminary Data, 26 November 2020, $<$ https://www.gov.uk/government/publications/innova-lateral-flow-sars-cov-2-antigentest-accuracy-in-liverpool-pilot-preliminary-data-26-november-2020> (2020).

69 Peto, T. COVID-19: Rapid Antigen detection for SARS-CoV-2 by lateral flow assay: a national systematic evaluation for mass-testing. medRxiv, 2021.2001.2013.21249563, doi:10.1101/2021.01.13.21249563 (2021).

70 Tu, Y. P. et al. Swabs Collected by Patients or Health Care Workers for SARS-CoV-2 Testing. N Engl J Med 383, 494-496, doi:10.1056/NEJMc2016321 (2020).

71 Lennard, Y. et al. An observational study of SARS-CoV-2 infectivity by viral load and demographic factors and the utility lateral flow devices to prevent transmission. URL http://modmedmicro. nsms. ox. ac. uk/wpcontent/uploads/2021/01/infectivity_manuscript_20210119_merged. pdf (2021).

72 Sabino, E. C. et al. Resurgence of COVID-19 in Manaus, Brazil, despite high seroprevalence. Lancet 397, 452-455, doi:10.1016/S0140-6736(21)00183-5 (2021).

73 Madhi, S. A. et al. Efficacy of the ChAdOx1 nCoV-19 Covid-19 Vaccine against the B.1.351 Variant. N Engl J Med, doi:10.1056/NEJMoa2102214 (2021).

74 Shinde, V. et al. Preliminary Efficacy of the NVX-CoV2373 Covid-19 Vaccine Against the B.1.351 Variant. medRxiv, 2021.2002.2025.21252477, doi:10.1101/2021.02.25.21252477 (2021).

75 Irwin, A. What it will take to vaccinate the world against COVID-19. Nature 592, 176178 (2021).

76 Davies, N. G. et al. Estimated transmissibility and impact of SARS-CoV-2 lineage B.1.1.7 in England. Science 372, doi:10.1126/science.abg3055 (2021).

77 Hosmer, D. W. \& Lemeshow, S. Goodness of fit tests for the multiple logistic regression model. Communs Statist 9, 1043-1069 (1980).

78 Janková, J., Shah, R. D., Bühlmann, P. \& Samworth, R. J. Goodness-of-fit testing in high dimensional generalized linear models. J. Roy. Statist. Soc., Ser. B 82, 773-795 (2020).

79 Enright, J. et al. SARS-CoV-2 infection in UK university students: Lessons from September-December 2020 and modelling insights for future student return. INI Preprint Series (2021). <https://www.newton.ac.uk/files/preprints/ni20004.pdf>. 
1143 Supplementary Table 1. Characteristics of study population.

\begin{tabular}{|c|c|c|c|c|c|}
\hline & & Eligible & Participating & Not-participating & $P$ value \\
\hline $\mathbf{N}$ & & 15,561 & 12,781 & 2,780 & \\
\hline \multirow[t]{3}{*}{ Sex } & Male & $8,060(51.8 \%)$ & $6,504(50.9 \%)$ & $1,556(56.0 \%)$ & \multirow[t]{3}{*}{$<0.001$} \\
\hline & Female & $7,213(46.4 \%)$ & $6,125(47.9 \%)$ & $1,088(39.1 \%)$ & \\
\hline & Other/unknown & $288(1.9 \%)$ & $152(1.2 \%)$ & $136(4.9 \%)$ & \\
\hline $\begin{array}{l}\text { Age, years, } \\
\text { median (IQR, } \\
\text { range) }\end{array}$ & & $\begin{array}{c}20.6(19.4-22.6, \\
16.4-71.7)\end{array}$ & $\begin{array}{c}20.5(19.3-22.3, \\
16.4-58.1)\end{array}$ & $\begin{array}{c}21.3(19.8-24.3, \\
17.4-71.7)\end{array}$ & $<0.001$ \\
\hline \multirow[t]{3}{*}{ Ethnicity } & White & $9,453(60.7 \%)$ & $8,168(63.9 \%)$ & $1,285(46.2 \%)$ & \multirow[t]{3}{*}{$<0.001$} \\
\hline & $\begin{array}{l}\text { Black, Asian and } \\
\text { minority ethnic }\end{array}$ & $5,302(34.1 \%)$ & $4,097(32.1 \%)$ & $1,205(43.3 \%)$ & \\
\hline & Refused/unknown & $806(5.2 \%)$ & $516(4.0 \%)$ & $290(10.4 \%)$ & \\
\hline \multirow[t]{3}{*}{ Residency } & UK & $9,870(63.4 \%)$ & $8,504(66.5 \%)$ & $1,366(49.1 \%)$ & \multirow[t]{3}{*}{$<0.001$} \\
\hline & International & $5,496(35.3 \%)$ & $4,205(32.9 \%)$ & $1,291(46.4 \%)$ & \\
\hline & Unknown & $195(1.3 \%)$ & $72(0.6 \%)$ & $123(4.4 \%)$ & \\
\hline \multirow[t]{6}{*}{ Year of study } & $1^{\text {st }}$ & $3,788(24.3 \%)$ & $3,336(26.1 \%)$ & $452(5.5 \%)$ & \multirow[t]{6}{*}{$<0.001$} \\
\hline & $2^{\text {nd }}$ & $3,239(20.8 \%)$ & $2,673(20.9 \%)$ & $566(20.4 \%)$ & \\
\hline & $3^{\text {rd }}$ & $2,832(18.2 \%)$ & $2,434(19.0 \%)$ & $398(14.3 \%)$ & \\
\hline & $4^{\text {th }}$ (or above) & $997(6.4 \%)$ & $844(6.6 \%)$ & $153(5.5 \%)$ & \\
\hline & Postgraduate & $4,369(28.1 \%)$ & $3,366(26.3 \%)$ & $1,003(36.0 \%)$ & \\
\hline & Missing & $336(2.2 \%)$ & $126(1.0 \%)$ & $210(7.5 \%)$ & \\
\hline \multirow[t]{3}{*}{ Stage } & Undergraduate & $10,857(69.8 \%)$ & $9,288(72.7 \%)$ & $1,569(56.4 \%)$ & \multirow[t]{3}{*}{$<0.001$} \\
\hline & Postgraduate & $4,369(28.1 \%)$ & $3,368(26.3 \%)$ & $1,001(36.0 \%)$ & \\
\hline & Missing & $335(2.2 \%)$ & $125(1.0 \%)$ & $210(7.5 \%)$ & \\
\hline \multirow[t]{5}{*}{ Course* } & $\begin{array}{l}\text { Undergraduate arts } \\
\text { and humanities }\end{array}$ & $5,490(35.3 \%)$ & $4,553(35.6 \%)$ & $937(33.7 \%)$ & \multirow[t]{5}{*}{$<0.001$} \\
\hline & $\begin{array}{l}\text { Undergraduate } \\
\text { science and } \\
\text { technology }\end{array}$ & $5,454(35.1 \%)$ & $4,806(37.6 \%)$ & $648(23.3 \%)$ & \\
\hline & $\begin{array}{l}\text { Postgraduate } \\
\text { vocational }\end{array}$ & $466(3.0 \%)$ & $343(2.7 \%)$ & $123(4.4 \%)$ & \\
\hline & Postgraduate (other) & $3,815(24.5 \%)$ & $2,953(23.1 \%)$ & $862(31.0 \%)$ & \\
\hline & Unknown & $336(2.2 \%)$ & $126(1.0 \%)$ & $210(7.6 \%)$ & \\
\hline \multirow[t]{4}{*}{ Household size } & 1 person & $472(3.0 \%)$ & $299(2.3 \%)$ & $173(6.2 \%)$ & \multirow[t]{4}{*}{$<0.001$} \\
\hline & 2 to 5 people & $4,535(29.1 \%)$ & $3,703(29.0 \%)$ & $832(29.9 \%)$ & \\
\hline & 6 to 10 people & $9,228(59.3 \%)$ & $7,688(60.2 \%)$ & $1,540(55.4 \%)$ & \\
\hline & $>10$ people & $1,326(8.5 \%)$ & $1,091(8.5 \%)$ & $235(8.5 \%)$ & \\
\hline
\end{tabular}

Student characteristics associated with programme participation were assessed by comparing participating and non-participating students using unpaired 2 sample 2 tailed $t$ tests (continuous variables) or chi-square tests (categorical variables).

${ }^{*}$ Courses are grouped as: undergraduate arts and humanities (undergraduate students in the School of Arts and Humanities and the School of Humanities and Social Sciences); undergraduate science and technology (undergraduate students in the School of Biological Sciences, the School of Physical Sciences and the School of Technology); postgraduate vocational courses (students in clinical medicine, clinical veterinary medicine and postgraduate certificates in education); and other postgraduate courses (all other postgraduate students, including those in doctoral and masters programmes). 
Supplementary Table 2. Tests conducted.

1159

\begin{tabular}{|c|c|c|c|c|c|c|c|c|c|c|c|c|c|}
\hline \multirow[t]{2}{*}{ Week } & \multirow[t]{2}{*}{ Dates } & \multirow[t]{2}{*}{$\begin{array}{c}\text { Eligible } \\
\text { students }\end{array}$} & \multirow[t]{2}{*}{$\begin{array}{l}\text { Participating } \\
\text { students }\end{array}$} & \multicolumn{8}{|c|}{ Asymptomatic screening programme } & \multicolumn{2}{|c|}{$\begin{array}{c}\text { University } \\
\text { symptomatic } \\
\text { testing }\end{array}$} \\
\hline & & & & $\begin{array}{l}\text { Screening } \\
\text { tests }\end{array}$ & $\begin{array}{l}\text { Valid } \\
\text { results }\end{array}$ & Scale* $^{*}$ & $\begin{array}{c}\text { Mean } \\
\text { swab } \\
\text { count per } \\
\text { pool }\end{array}$ & $\begin{array}{c}\text { Students } \\
\text { screened } \\
\text { (estimated) }\end{array}$ & $\begin{array}{c}\text { Individual } \\
\text { confirmatory } \\
\text { tests }\end{array}$ & $\begin{array}{l}\text { Valid } \\
\text { results }\end{array}$ & $\begin{array}{c}\text { Students } \\
\text { screened } \\
\text { per total } \\
\text { tests }\end{array}$ & $\begin{array}{l}\text { Individual } \\
\text { tests }\end{array}$ & $\begin{array}{l}\text { Valid } \\
\text { results }\end{array}$ \\
\hline 1 & 5 Oct -11 Oct & 15,479 & 11,638 & 1,867 & 1,837 & $\begin{array}{c}2 \text { students } \\
\text { per pool }\end{array}$ & 1.87 & 3,435 & 36 & 34 & 1.81 & 102 & 96 \\
\hline 2 & 12 Oct -18 Oct & 15,511 & 12,100 & 1,890 & 1,866 & $\begin{array}{c}2 \text { students } \\
\text { per pool }\end{array}$ & 1.92 & 3,583 & 56 & 56 & 1.84 & 221 & 219 \\
\hline 3 & 19 Oct -25 Oct & 15,488 & 12,195 & 1,913 & 1,886 & Half-pool & 2.47 & 4,658 & 105 & 104 & 2.31 & 195 & 194 \\
\hline 4 & 26 Oct -1 Nov & 15,440 & 12,383 & 1,923 & 1,900 & Half-pool & 2.92 & 5,548 & 109 & 109 & 2.73 & 109 & 109 \\
\hline 5 & $2 \mathrm{Nov}-8 \mathrm{Nov}$ & 15,385 & 12,372 & 1,873 & 1,865 & Half-pool & 2.49 & 4,644 & 79 & 77 & 2.38 & 112 & 110 \\
\hline 6 & $9 \mathrm{Nov}-15 \mathrm{Nov}$ & 15,323 & 12,350 & 1,864 & 1,851 & Half-pool & 2.89 & 5,349 & 228 & 226 & 2.56 & 265 & 263 \\
\hline 7 & $16 \mathrm{Nov}-22 \mathrm{Nov}$ & 15,307 & 12,424 & 1,743 & 1,727 & Half-pool & 2.42 & 4,179 & 102 & 102 & 2.27 & 87 & 87 \\
\hline 8 & $23 \mathrm{Nov}-29 \mathrm{Nov}$ & 15,309 & 12,498 & 1,919 & 1,889 & Whole pool & 5.02 & 9,483 & 45 & 44 & 4.83 & 28 & 28 \\
\hline 9 & $30 \mathrm{Nov}-6 \mathrm{Dec}$ & 15,310 & 12,544 & 1,953 & 1,938 & Whole pool & 4.90 & 9,496 & 52 & 52 & 4.74 & 27 & 27 \\
\hline $\begin{array}{c}\text { Weeks } \\
1-2\end{array}$ & 5 Oct -18 Oct & & & 3,757 & 3,703 & $\begin{array}{c}2 \text { students } \\
\text { per pool }\end{array}$ & & 7,018 & 92 & 90 & 1.82 & 323 & 315 \\
\hline $\begin{array}{c}\text { Weeks } \\
3-7\end{array}$ & 19 Oct $-22 \mathrm{Nov}$ & & & 9,316 & 9,229 & Half-pool & & 24,379 & 623 & 618 & 2.45 & 768 & 763 \\
\hline $\begin{array}{c}\text { Weeks } \\
8-9\end{array}$ & $23 \mathrm{Nov}-6 \mathrm{Dec}$ & & & 3,872 & 3,827 & Whole pool & & 18,979 & 97 & 96 & 4.78 & 55 & 55 \\
\hline $\begin{array}{c}\text { All } \\
\text { weeks }\end{array}$ & 5 Oct -6 Dec & 15,561 & 12,979 & 16,945 & 16,759 & & & 50,376 & 812 & 804 & 2.84 & 1,146 & 1,133 \\
\hline
\end{tabular}

1161 *Phase 1 (weeks 1-2): two students per testing pool screened each week; phase 2 (weeks 3-7): half of each testing pool screened on alternating week; phase

11623 (weeks 8-9): whole testing pool screened each week. 


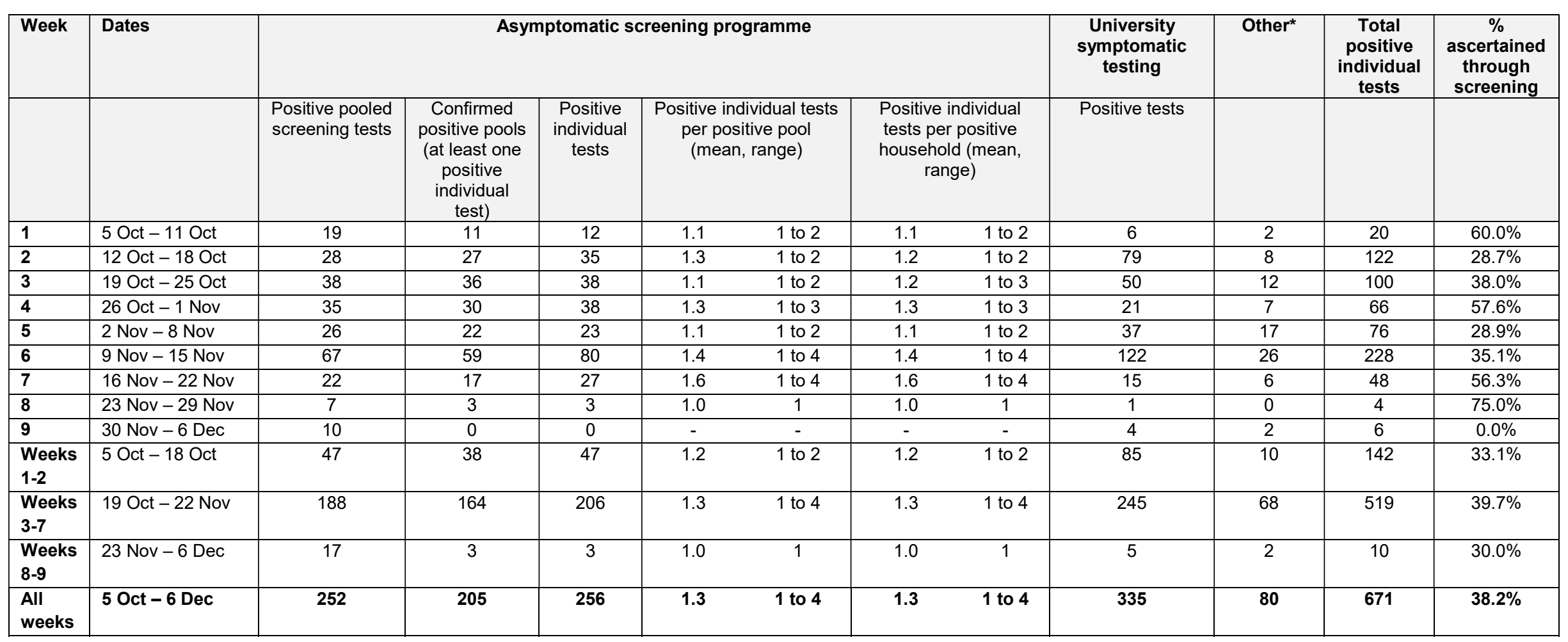

1167

1168

1169

1170

1171
${ }^{*}$ Other includes: positive results obtained through other testing pathways, such as NHS testing facilities, and reported to the university by Public Health

England; and 7 asymptomatic positive cases identified through a distinct programme of screening undertaken by the university in conjunction with local public health teams during an outbreak investigation in a single accommodation block during term week 3 . An additional 2 cases reported to the university by Public Health England during the study period were excluded, because their test dates could not be confirmed. 
1173 Supplementary Table 4. Technical validation: number of replicates detected for each 1174 target concentration of SARS-CoV-2 virus.

1175

\begin{tabular}{|l|c|c|c|c|c|}
\hline $\begin{array}{c}\text { Target concentration } \\
\text { of SARS CoV-2 } \\
\text { (dC/mL) }\end{array}$ & $\begin{array}{c}\text { Calculated } \\
\text { SARS Cov-2 } \\
\text { copies per RT- } \\
\text { PCR reaction }\end{array}$ & $\begin{array}{c}\text { Number of } \\
\text { swabs }\end{array}$ & $\begin{array}{c}\text { Replicates } \\
\text { detected }\end{array}$ & $\begin{array}{c}\text { Number of } \\
\text { swabs }\end{array}$ & $\begin{array}{c}\text { Replicates } \\
\text { detected }\end{array}$ \\
\hline 250,000 & 4,444 & 1 & $3 / 3$ & $5-6$ & $3 / 3$ \\
\hline 100,000 & 1,778 & 1 & $3 / 3$ & $5-7$ & $3 / 3$ \\
\hline 25,000 & 444 & 1 & $2 / 2$ & $5-6$ & $3 / 3$ \\
\hline 10,000 & 178 & 1 & $3 / 3$ & $5-6$ & $3 / 3$ \\
\hline 2,500 & 44 & 1 & $3 / 3$ & $5-6$ & $3 / 3$ \\
\hline 1,000 & 18 & 1 & $3 / 3$ & $5-7$ & $3 / 3$ \\
\hline 500 & 9 & 1 & $3 / 3$ & $5-6$ & $3 / 3$ \\
\hline 250 & 4 & 1 & $3 / 3$ & $5-8$ & $3 / 3$ \\
\hline 125 & 2 & 1 & $3 / 3$ & $5-7$ & $3 / 3$ \\
\hline
\end{tabular}


1177 Supplementary Table 5. Distribution of CT values for individual confirmatory tests.

1178

\begin{tabular}{|c|c|c|c|c|c|c|}
\hline & & & CT value & & & \\
\hline & $<20$ & $20-25$ & $25-30$ & $30-35$ & $\geq 35$ & All \\
\hline Symptomatic testing & $60(18 \%)$ & $137(41 \%)$ & $83(25 \%)$ & $41(12 \%)$ & $14(4 \%)$ & $335(100 \%)$ \\
\hline $\begin{array}{l}\text { Asymptomatic screening } \\
\text { programme }\end{array}$ & $23(9 \%)$ & $85(33 \%)$ & $68(27 \%)$ & $43(17 \%)$ & $37(14 \%)$ & $256(100 \%)$ \\
\hline $\begin{array}{l}\text { Presymptomatic with } \\
\text { cardinal symptoms }\end{array}$ & $6(9 \%)$ & $30(44 \%)$ & $21(31 \%)$ & $7(10 \%)$ & $6(9 \%)$ & $68(100 \%)$ \\
\hline Minor symptoms & $4(9 \%)$ & $14(33 \%)$ & $17(40 \%)$ & $3(7 \%)$ & $5(12 \%)$ & $43(100 \%)$ \\
\hline Asymptomatic & $2(7 \%)$ & $6(21 \%)$ & $4(14 \%)$ & $7(24 \%)$ & $10(34 \%)$ & $29(100 \%)$ \\
\hline
\end{tabular}

1179 
Supplementary Table 6. Single variable logistic regression analysis of student characteristics associated with a positive SARS-CoV-2 result, $n=12,781$.

1182

\begin{tabular}{|c|c|c|c|c|c|c|}
\hline Variable & Category & Positive & $\begin{array}{c}\text { Not } \\
\text { positive }\end{array}$ & OR & $(95 \% \mathrm{Cl})$ & $P$ value \\
\hline \multirow[t]{3}{*}{ Sex } & Female & 325 & 5,800 & & & \\
\hline & Male & 346 & 6,158 & 1.00 & $0.86-1.17$ & 0.97 \\
\hline & Other/unknown & 5 & 147 & 0.61 & $0.21-1.34$ & 0.28 \\
\hline \multirow[t]{3}{*}{ Ethnicity } & White & 509 & 7,659 & & & \\
\hline & Other & 153 & 3,944 & 0.58 & $0.48-0.70$ & $<0.001$ \\
\hline & Unknown & 14 & 502 & 0.42 & $0.23-0.69$ & 0.002 \\
\hline \multirow[t]{3}{*}{ Residency } & UK & 570 & 7,934 & & & \\
\hline & International & 103 & 4,102 & 0.35 & $0.28-0.43$ & $<0.001$ \\
\hline & Unknown & 3 & 69 & 0.61 & $0.15-1.63$ & 0.40 \\
\hline \multirow[t]{4}{*}{ Year of study } & $1^{\text {st }}$ & 367 & 5,195 & & & \\
\hline & $2^{\text {nd }}$ & 166 & 2,999 & 0.78 & $0.65-0.95$ & 0.01 \\
\hline & $3^{\text {rd }}$ or higher & 142 & 3,786 & 0.53 & $0.43-0.65$ & $<0.001$ \\
\hline & Unknown & 1 & 125 & 0.11 & $0.006-0.51$ & 0.03 \\
\hline \multirow[t]{5}{*}{ Course } & $\begin{array}{l}\text { Undergraduate arts } \\
\text { and humanities }\end{array}$ & 348 & 4,205 & & & \\
\hline & $\begin{array}{l}\text { Undergraduate } \\
\text { science and } \\
\text { technology }\end{array}$ & 246 & 4,560 & 0.65 & $0.55-0.77$ & $<0.001$ \\
\hline & $\begin{array}{l}\text { Postgraduate } \\
\text { vocational }\end{array}$ & 22 & 321 & 0.83 & $0.52-1.26$ & 0.41 \\
\hline & Postgraduate (other) & 59 & 2,894 & 0.25 & $0.18-0.32$ & $<0.001$ \\
\hline & Unknown & 1 & 125 & 0.10 & $0.0055-0.43$ & 0.02 \\
\hline \multirow{5}{*}{$\begin{array}{l}\text { Household } \\
\text { size }\end{array}$} & Minimum & 1 & 1 & 1.08 & $1.05-1.11$ & $<0.001$ \\
\hline & $25^{\text {th }}$ centile & 6 & 5 & & & \\
\hline & Median & 7 & 7 & & & \\
\hline & $75^{\text {th }}$ centile & 8 & 8 & & & \\
\hline & Maximum & 20 & 20 & & & \\
\hline
\end{tabular}

1183 
Supplementary Table 7. Multivariable logistic regression analysis of student characteristics associated with a positive SARS-CoV-2 result, $n=12,781$.

1186

1187

\begin{tabular}{|c|c|c|c|c|}
\hline Variable & Category & OR & $95 \% \mathrm{Cl}$ & $P$ value \\
\hline \multirow[t]{3}{*}{ Sex } & Female & & & \\
\hline & Male & 1.21 & $1.03-1.42$ & 0.02 \\
\hline & Other/unknown & 0.44 & $0.07-1.42$ & 0.26 \\
\hline \multirow[t]{3}{*}{ Ethnicity } & White & & & \\
\hline & Other & 0.69 & $0.57-0.84$ & $<0.001$ \\
\hline & Unknown & 0.46 & $0.23-0.81$ & 0.01 \\
\hline \multirow[t]{3}{*}{ Residency } & UK & & & \\
\hline & International & 0.53 & $0.42-0.67$ & $<0.01$ \\
\hline & Unknown & 12.4 & $1.63-113$ & 0.01 \\
\hline \multirow[t]{4}{*}{ Year of study } & $1^{\text {st }}$ & & & \\
\hline & $2^{\text {nd }}$ & 0.63 & $0.52-0.76$ & $<0.001$ \\
\hline & $3^{\text {rd }}$ or higher & 0.44 & $0.36-0.54$ & $<0.001$ \\
\hline & Unknown & 0.04 & $0.002-0.24$ & 0.004 \\
\hline \multirow[t]{5}{*}{ Course } & $\begin{array}{l}\text { Undergraduate arts and } \\
\text { humanities }\end{array}$ & & & \\
\hline & $\begin{array}{l}\text { Undergraduate science and } \\
\text { technology }\end{array}$ & 0.74 & $0.62-0.88$ & 0.001 \\
\hline & Postgraduate vocational & 0.73 & $0.45-1.11$ & 0.16 \\
\hline & Postgraduate (other) & 0.28 & $0.21-0.38$ & $<0.001$ \\
\hline & Unknown & NA & - & - \\
\hline Household size & & 1.06 & $1.03-1.09$ & $<0.001$ \\
\hline
\end{tabular}


Supplementary Table 8: Model settings, parameters, and assumptions.

\begin{tabular}{|c|c|c|c|}
\hline Parameter or setting & Value or assumption & Source & Change from HE-INI model $^{1}$ \\
\hline \multicolumn{4}{|l|}{ Network settings } \\
\hline Number of individuals & 12,781 & From data: number of study participants & Yes - generic 15,000 used in preprint \\
\hline Household structure & $\begin{array}{l}\text { Full specification: } \\
\text { \{1: } 0.151,2: 0.105,3: 0.074,4: 0.114,5: 0.115, \\
\text { 6:0.130, } 7: 0.094,8: 0.117,9: 0.036,10: 0.029, \\
\text { 11:0.014, 12:0.012, 13:0.003, 14:0.003, } \\
\text { 15:0.001, 16:0.001, 18:0.001\} } \\
\text { This notation means: } \\
\text { Size_of_household:fraction_of_households_t } \\
\text { hat_size }\end{array}$ & $\begin{array}{l}\text { From data: household structure of eligible } \\
\text { students }\end{array}$ & $\begin{array}{l}\text { Yes }-\{10: 0.5,5: 0.5\} \text { used in preprint, } \\
\text { arbitrary choice }\end{array}$ \\
\hline Sizes of other activity groups & $\begin{array}{l}\text { Full specification: } \\
\{50: 0.01,10: 0.5,5: 0.49\} \\
\\
\text { Notation as above }\end{array}$ & $\begin{array}{l}\text { By assumption: to model a majority of small } \\
\text { groups, with a small number of larger events }\end{array}$ & $\begin{array}{l}\text { Yes - }\{40: 0.05,10: 0.3,5: 0.5,3: 0.15\} \\
\text { used in preprint, arbitrary choice }\end{array}$ \\
\hline Number of other activity groups & 4,000 & $\begin{array}{l}\text { By assumption: note relationship to probability of } \\
\text { transmission for a non-household contact below }\end{array}$ & $\begin{array}{l}\text { Yes }-3,000 \text { used in preprint, arbitrary } \\
\text { choice }\end{array}$ \\
\hline \multicolumn{4}{|l|}{ Disease progression and transmission } \\
\hline $\begin{array}{l}\text { Probability of transmission for a } \\
\text { household contact per day }\end{array}$ & 0.017 & $\begin{array}{l}\text { From data: calibrated to produce the observed } \\
\text { within-household attack rate, accounting for } \\
\text { symptomatic and asymptomatic infections }\end{array}$ & $\begin{array}{l}\text { Yes }-0.025 \text { used in preprint, chosen for } \\
\text { plausibility }\end{array}$ \\
\hline $\begin{array}{l}\text { Ratio of probability of transmission for a } \\
\text { non-household contact per day to } \\
\text { probability of transmission for a } \\
\text { household contact per day }\end{array}$ & 1.0 & $\begin{array}{l}\text { From data: calibrated to produce a between- } \\
\text { household attack rate roughly consistent with the } \\
\text { observed rate to week } 7 \text { (before the UK national } \\
\text { lockdown) } \\
\text { Note that this parameter has a relationship to the } \\
\text { sizes and number of other activity groups: an } \\
\text { increase in the number of other activity groups or } \\
\text { their sizes gives similar results to an increase in } \\
\text { this parameter } \\
\text { For computational reasons it is more efficient to } \\
\text { increase this parameter than the total number of } \\
\text { non-household contacts }\end{array}$ & $\begin{array}{l}\text { Yes }-0.2 \text { used in preprint, chosen for } \\
\text { plausibility }\end{array}$ \\
\hline Mean non-infectious latent period & 2.9 days & Alene et al. $(2021)^{2}$ & $\begin{array}{l}\text { Yes (small change) - } 3 \text { days in preprint, } \\
\text { chosen for plausibility }\end{array}$ \\
\hline
\end{tabular}




\begin{tabular}{|c|c|c|c|}
\hline Mean presymptomatic infectious period & 3.6 days & $\begin{array}{l}\text { From data: observed presymptomatic infectious } \\
\text { period }\end{array}$ & $\begin{array}{l}\text { Yes }-2 \text { days in preprint, chosen for } \\
\text { plausibility }\end{array}$ \\
\hline Proportion asymptomatic & $19.7 \%$ & $\begin{array}{l}\text { From data: observed proportion of asymptomatic } \\
\text { students }\end{array}$ & Yes $-50 \%$, chosen for plausibility \\
\hline $\begin{array}{l}\text { Infectiousness of presymptomatics } \\
\text { relative to symptomatics }\end{array}$ & Equal & By assumption & No change \\
\hline $\begin{array}{l}\text { Infectiousness of asymptomatics relative } \\
\text { to symptomatics }\end{array}$ & $18 \%$ & $\begin{array}{l}\text { From data: observed ratio of secondary household } \\
\text { attack rates for asymptomatic and symptomatic } \\
\text { index cases }\end{array}$ & $\begin{array}{l}\text { Yes - equal infectiousness used in } \\
\text { preprint, chosen for plausibility }\end{array}$ \\
\hline $\begin{array}{l}\text { Mean infectious period after symptoms } \\
\text { develop }\end{array}$ & 4 days & Singanayagam, A. et al. $(2020)^{3}$ & $\begin{array}{l}\text { Yes }-10 \text { days used in preprint, chosen } \\
\text { for plausibility }\end{array}$ \\
\hline $\begin{array}{l}\text { Total mean period of infectiousness of an } \\
\text { asymptomatic individual }\end{array}$ & $\begin{array}{l}\text { Equal to symptomatic individual (mean } 7.6 \\
\text { days) }\end{array}$ & By assumption & No change \\
\hline \multicolumn{4}{|l|}{ Testing and isolation } \\
\hline $\begin{array}{l}\text { Probability and speed of symptomatic } \\
\text { tests }\end{array}$ & $\begin{array}{l}\text { Symptomatic individuals immediately seek } \\
\text { tests with no false positives or false } \\
\text { negatives. Positive tests result in household } \\
\text { isolation from the following day }\end{array}$ & By assumption & No change \\
\hline Participation in asymptomatic screening & $100 \%$ & By assumption & No change \\
\hline Performance of asymptomatic tests & $\begin{array}{l}\text { Asymptomatic testing is perfect with no false } \\
\text { negatives or false positives. Positive tests } \\
\text { result in household isolation from the } \\
\text { following day }\end{array}$ & By assumption & No change \\
\hline Isolation & $\begin{array}{l}\text { Entire household isolates upon any positive } \\
\text { test within the household }\end{array}$ & By assumption & No change \\
\hline Expected isolation period & 14 days & By assumption & No change \\
\hline Isolation implications for contact & $\begin{array}{l}\text { When a household isolates, all contacts } \\
\text { between members of the household and non- } \\
\text { members cease, but all contacts within the } \\
\text { household continue as before including with } \\
\text { the index case. }\end{array}$ & By assumption & No change \\
\hline Tracing & $\begin{array}{l}50 \% \text { probability of each non-household } \\
\text { contact of a test-positive being contacted and } \\
\text { isolating from the day after the positive test. }\end{array}$ & By assumption & No change \\
\hline
\end{tabular}

1. Enright, J. et al. SARS-CoV-2 infection in UK university students: Lessons from September-December 2020 and modelling insights for future student return. INI Preprint Series (2021). Available in preprint at: https://www.newton.ac.uk/files/preprints/ni20004.pdf.

2. Alene, M. et al. Serial interval and incubation period of COVID-19: a systematic review and meta-analysis. BMC Infect Dis 21, 257, doi:10.1186/s12879-021-05950-x (2021).

3. Singanayagam, A. et al. Duration of infectiousness and correlation with RT-PCR cycle threshold values in cases of COVID-19, England, January to May 2020. Euro Surveill 25, doi:10.2807/1560-7917.ES.2020.25.32.2001483 (2020). 


\section{Rapid Response Working Group to Test, Trace and Isolate}

Patrick Maxwell, Nicholas Matheson, Chris Abell, Vickie Braithwaite, Craig Brierley, Jon Crowcroft, Aastha Dahal, Kathryn Faulkner, Michael Glover, lan Goodfellow, Jane Greatorex, Jon Holgate, Rob Howes, Laura James, Paul Lehner, Ian Leslie, Kathleen Liddell, Ben Margolis, Duncan McFarlane, Sally Morgan, Linda Sheridan, Sally Valletta, Anna Vignoles, Martin Vinnell, Ben Warne, Michael

\section{Clinical Research Nurse Team}

Stewart Fuller, Marina Metaxaki, Sarah Hilborne, Sarah Berry, Mahin Bagheri Kahkeshi, Dawn Hancock, Jennifer Winster

\section{Biopharma RD, AstraZeneca, Cambridge}

Rob Howes, Alexandra Orton, Julie Douthwaite, Steve Rees

Charles River Laboratories, Chesterford Research Park, Saffron Walden, UK

Christopher Brown, Daniel R Jones, Fred Kuenzi, Jennifer Rankin, Ian Waddell

\section{Statistics/modelling}

Jessica Enright, Richard Samworth

\section{University Information Services}

Jon Holgate, Vijay Samtani, Gabriela Ahmadi-Assalemi, Tom Feather, Robin Goodall, Steve Hoensch, Dean Johnson, Martin Hunt, Nick Mathieson, Katya Nikitina, Zara Sheldrake

\section{Clinical School Computing Service}

Martin Keen, Aris Sato, David Connor, Jonathan Tolhurst, Jack Williman

\section{Clinical School Information Governance Office}

Victoria Hollamby

\section{COVID-19 Operations Centre, University of Cambridge}

Gillian Weale, Sinead Jordan, Tania Fatseas, Peter Taylor, Christine Georgiou, Michelle Caspersz, Claire McNulty, Richard Davies

Institute for Manufacturing, University of Cambridge

Duncan McFarlane, Rebecca Clarke, Darius Danaei, Rory Dyer, Rob Glew, Oliver Lambson

\section{Testing Kit Packing Team}

Karen Gibbs, Barbara Mozdzen, Gabor Raub, Asako Radecki, Phil White, Robert Hughes, Lucie Gransden, Matt Ceaser

\section{Administrative Support}

Robert Sing, Karl Wilson

\section{Operational Systems Support}

Ajith Parlikad, Maharshi Dhada, Tom Ridgman 


\section{Collegiate University}

Andy Neely, Robert Henderson, Edna Murphy, Matthew Russell, Anthony Freeling, Steve Poppitt, Jo Tynan

\section{Data Protection and Information Compliance}

James Knapton, Filippo Marchetti

\section{COG-UK Cambridge}

Sharon Peacock, lan Goodfellow, Ewan Harrison, Dinesh Aggarwal, Thomas Fieldman, Beth Blane, Yasmin Chaudhry, Daniela De Angelis, Theresa Feltwell, Iliana Georgana, Nazreen F. Hadjirin, Grant Hall, William Hamilton, Myra Hosmillo, Chris Illingworth, Aminu Jahun, Danielle Leek, Catherine Ludden, Malte Pinckert

\section{Cambridge University Hospitals NHS Foundation Trust}

Ashley Shaw, Afzal Chaudhry, Nicholas M Brown, Lenette Mactavous, Sophie Hannan, Aleksandra Hosaja, Clare Leong

Occupational Health and Wellbeing (Cambridge University Hospitals NHS Foundation Trust)Jo 


\section{Supplementary Files}

This is a list of supplementary files associated with this preprint. Click to download.

- SupplementaryInformationWarneetal.pdf

- ExtendedFiguresHighResWarneetal.pdf

- MainFiguresHighResWarneetal.pdf 